ESAIM: PROCEEDINGS AND SURVEYS, March 2015, Vol. 50, p. 40-65

Franck BOYER, Thierry GALLOUET, Raphaèle HERBIN and Florence HUBERT Editors

\title{
NEW APPROACHES TO DESCRIBING ADMISSIBILITY OF SOLUTIONS OF SCALAR CONSERVATION LAWS WITH DISCONTINUOUS FLUX *,**
}

\author{
BORIS ANDREIANOV ${ }^{1}$
}

\begin{abstract}
Hyperbolic conservation laws of the form $u_{t}+\operatorname{div} \mathfrak{f}(t, x ; u)=0$ with discontinuous in $(t, x)$ flux function $f$ attracted much attention in last 20 years, because of the difficulties of adaptation of the classical Kruzhkov approach developed for the smooth case. In the discontinuous-flux case, non-uniqueness of mathematically consistent admissibility criteria results in infinitely many different notions of solution. A way to describe all the resulting $L^{1}$-contractive solvers within a unified approach was proposed in the work [Andreianov, Karlsen, Risebro, 2011]. We briefly recall the ideas and results developed there for the model one-dimensional case with $\mathfrak{f}(t, x ; u)=f_{l}(u) \mathbb{1}_{x<0}+f_{r}(u) \mathbb{1}_{x>0}$ and highlight the main hints needed to address the multi-dimensional situation with curved interfaces.

Then we discuss two recent developments in the subject which permit to better understand the issue of admissibility of solutions in relation with specific modeling assumptions; they also bring useful numerical approximation strategies. A new characterization of limits of vanishing viscosity approximation proposed in [Andreianov and Mitrović, 2014] permits to encode admissibility in singular but intuitively appealing entropy inequalities. Transmission maps introduced in ([Andreianov and Cancès, 2015]) have applications in modeling flows in strongly heterogeneous porous media and lead to a simple algorithm for numerical approximation of the associated solutions.

Moreover, in order to embed all the aforementioned results into a natural framework, we put forward the concept of interface coupling conditions (ICC) which role is analogous to the role of boundary conditions for boundary-value problems. We link this concept to known examples and techniques.
\end{abstract}

\section{INTRODUCTION}

Consider the Cauchy problem for scalar conservation laws under the general form

$$
\begin{gathered}
u_{t}+\operatorname{div} \mathfrak{f}(t, x ; u)=0, \\
\left.u\right|_{t=0}=u_{0}
\end{gathered}
$$

for $(t, x) \in(0,+\infty) \times \mathbb{R}^{N}, N \geq 1$. The main ideas that underlie the modern theory of (1) go back to Hopf, Oleïnik, Lax, Vol'pert and Kruzhkov (cf. the fundamental text of Dafermos [43]). These ideas achieved their classical form in the work [52], where the concept of Kruzhkov entropy solution was firmly established, supplied with a collection of well-posedness techniques.

The work [52] dealt with the case of sufficiently smooth dependence of $\mathfrak{f}$ on $(t, x ; u)$; at the present stage of the theory, well-posedness is achieved for Lipschitz in all variables flux $\mathfrak{f}$ (bounds derived from a maximum

\footnotetext{
* The author was supported by the French ANR project CoToCoLa

** The author is deeply indebted to Clément Cancès, Darko Mitrović and Karima Sbihi for the collaborations which are at the origin of results presented in this survey. He also thanks Nina Aguillon, Shyam S. Ghoshal, Cyril Imbert, Kenneth H. Karlsen, Régis Monneau, Nils Henrik Risebro and Nicolas Seguin for stimulating discussions.

1 Laboratoire de Mathématiques, CNRS UMR 6623, Université de Franche-Comté, Besançon, France

et Laboratoire J.-L. Lions CNRS UMR 7598, Université Paris 6, France
}

(C) EDP Sciences, SMAI 2015 
principle permit to include the case of local in $u$ Lipschitz regularity of $\mathfrak{f}$ ). An entropy solution of (1) is an $L^{\infty}$ function $u$ satisfying for all constant $k \in \mathbb{R}$,

$$
|u-k|_{t}+\operatorname{div}(\operatorname{sign}(u-k)(\mathfrak{f}(t, x ; u)-\mathfrak{f}(t, x ; k)))+\operatorname{sign}(u-k) \operatorname{div} \mathfrak{f}(t, x ; k) \leq 0
$$

in the sense of distributions, with appropriately taken into account initial conditions (see [52] and [60]).

In the special case of $(t, x)$-independent $\mathfrak{f}$, less regularity of $\mathfrak{f}$ in unknown $u$ is needed. Such fluxes $\mathfrak{f}$ can be included under the assumption of mere continuity if space dimension is equal to one, while Hölder continuity of order $1-1 / N$ is a sufficient condition for space dimension $N>1$; we refer to $[7,18,27,29,53]$ for this line of research, see also [65] for related results based on different techniques. Classical results concern $L^{\infty}$ or $L^{1} \cap L^{\infty}$ data; extensions to $L^{1}$ and to $L^{1}+L^{\infty}$ exploit the notions of kinetic ( [63] and references therein) and renormalized ( [28]) solutions.

Most of classical models based on (1) (such as the LWR vehicular traffic model, the Buckley-Leverett model for flows in porous media, etc.) lead to Lipschitz continuous f . Finer developments that require to deal with discontinuous fluxes $\mathfrak{f}$ attracted much attention in the two last decades. Discontinuity of $\mathfrak{f}$ in $u$ appears in some models with implicit constitutive relations. This was treated in slightly different ways in $[32,56]$ and in $[48]$; recently in [47], a combined approach was proposed in view of obtaining well-posedness results for $L^{1} \cap L^{\infty}$ data under the least restrictive assumptions of $\mathfrak{f}$. The techniques of the $u$-discontinuous case are based upon a monotone, possibly multivalued change of unknown that permits to exploit the original techniques of [52] or the technique of [65]; as a result, the authors obtain a unique "entropy solution" semigroup that inherits the $L^{1}$ contraction property known from the case of Lipschitz flux.

The situation is drastically different when it comes to the case of $(t, x)$-discontinuous flux $\mathfrak{f}$. In the sequel, we will always assume (at least local) Lipschitz continuity of $\mathfrak{f}$ in $u$, but concentrate on the difficulties coming from $x$ or $(t, x)$-discontinuity ${ }^{1}$.

Except for $\S 1.3$ and $\S 2.3$, we will limit our attention to the model one-dimensional problem with

$$
\mathfrak{f}(t, x ; u)=f_{l}(u) \mathbb{1}_{x<0}+f_{r}(u) \mathbb{1}_{x>0} .
$$

The Cauchy problem for the evolution equation (1) with the flux $\mathfrak{f}$ of the form (4) has been addressed in a number of works; let us refer to [15] and references therein for analysis of well-posedness of the Cauchy problem $(1),(4),(2)$. Let us only say that there is no hope to "uniquely extend" the Kruzhkov theory to the $x$-discontinuous flux case. Indeed, the key feature of this problem, first observed by Adimurthi, Mishra and Veerappa Gowda in [3], is the following:

$$
\text { there exist infinitely many } L^{1} \text {-contractive "entropy solution" semigroups for (1),(4). }
$$

In practice, each of these solvers can be characterized by some distinctive admissibility condition imposed on solutions; we further discuss this issue in the sequel, starting from $\S 1.1$. Actually, these different solvers (solution semigroups) may correspond to different modeling assumptions, cf. the work of $\mathrm{C}$. Cancès and the author [9]. One sees in [9] that some kind of interface coupling should be explicitly prescribed for ensuring well-posedness of (1),(4),(2), these conditions being a part of the model one wishes to consider. Making explicit such coupling conditions is also mandatory for its consistent numerical approximation and most importantly, for the identification of the underlying modelling assumptions.

\footnotetext{
${ }^{1}$ Let us observe in passing that in $[47,49]$ the authors established existence and uniqueness of entropy solutions with a combination of $u$ - and $x$-discontinuities; this approach is based on the particular interpretation [62] of problems with $x$-discontinuous flux, which corresponds to a praticular choice of interface coupling for models of kind (1),(4). As we stress below, there are infinitely many different options for the choice of interface coupling. The one of $[47,49]$ is of particular interest because it describes the vanishing viscosity limits, that are recognized as physically correct solutions in many (but not in all) applications.
} 
Let us point out an elementary, although somewhat unexpected - at a first glance ${ }^{2}$ - analogy between problems (1) with piecewise continuous in $(t, x)$ flux and much better studied boundary-value problems. Indeed, the situation of non-uniqueness (NU) can be compared with the well known situation for the Laplace equation in a bounded regular domain $\Omega$ of $\mathbb{R}^{N}$ with exterior unit normal $\nu$. Namely, the problem

$$
u-\Delta u=f \text { in } \Omega
$$

has infinitely many $L^{1}$-contractive solution maps $S_{\beta}: f \mapsto u$ (defined as variational or as renormalized solutions, according to the integrability of $f$, see, e.g., [23]) that correspond to different boundary conditions (BC, for short) which can take the general form

$$
\mathcal{H}^{N-1} \text {-a.e. on } \partial \Omega, \quad\left(\left(\gamma_{\text {sol }} u\right)(t),\left(\gamma_{\text {flux. } \nu}(\mathcal{F} \cdot \nu)\right)(t)\right) \in \beta
$$

Here $\beta$ (called BC graph in the sequel) is a maximal monotone graph in $\mathbb{R} \times \mathbb{R} ; \mathcal{F}:=-\nabla u$ is the flux governing the distribution of $u$ in (5); and $\gamma_{\text {sol }}, \gamma_{\text {flux. } \nu}$ are the appropriately defined trace operators. For example, the family of Robin boundary conditions $\lambda u+(1-\lambda) \frac{\partial u}{\partial \nu}=0$ with different values of $\lambda \in(0,1)$ corresponds to the family of BC graphs $\beta=\left\{\left(z, \frac{\lambda}{1-\lambda} z\right) \mid z \in \mathbb{R}\right\}$; the Neumann condition corresponds to the BC graph $\beta=\mathbb{R} \times\{0\}$, while the Dirichlet condition corresponds to the multivalued BC graph which can be described by $\beta=\{0\} \times \mathbb{R}$. Further boundary conditions of the above type may be of interest in applications, such as obstacle conditions ${ }^{3}$. The situation remains almost the same if the boundary-value problem for the Laplace equation (5) is replaced with the initial-boundary value problem (IBVP, for short) for the scalar conservation law $u_{t}+\operatorname{div} \mathcal{F}=0$ where $\mathcal{F}=\mathfrak{f}(u)$. The main difference here is that the BC graph $\beta$ has to be appropriately projected in order to ensure well-posedness of the problem (we refer to [21], which main idea and result will be briefly recalled in the sequel).

In this note, we claim that the multiplicity of solvers comes from the fact that the discontinuity surface $(\{(t, x) \mid t>0, x=0\}$ for the flux $\mathfrak{f}$ given by $(4))$, called interface in the sequel, plays the role of an embedded boundary. We put forward the fact that different $L^{1}$-contractive solution semigroups in (NU) actually correspond to different choices of interface coupling conditions for problem (1),(4). The latter can be expressed mimicking the formalism of "dissipative boundary conditions" exploited in the work [21] of K. Sbihi and the author. Namely, one asks (formally) that the traces

$$
u_{l}(t):=\lim _{x \rightarrow 0^{-}} u(t, x), u_{r}(t):=\lim _{x \rightarrow 0^{+}} u(t, x)
$$

of an admissible solution $u$ and the associated traces of the normal to the interface flux

$$
F_{l}(t):=\lim _{x \rightarrow 0^{-}} f_{l}(u(t, x)), \quad F_{r}(t):=\lim _{x \rightarrow 0^{+}}-f_{r}(u(t, x))
$$

verify for a.e. $t>0$

$$
\left(u_{l}(t), u_{r}(t) ; F_{l}(t), F_{r}(t)\right) \in \mathcal{H}
$$

for some subset $\mathcal{H}$ of $\mathbb{R}^{2} \times \mathbb{R}^{2}$ verifying an appropriate "maximal monotonicity" condition (see Section 4 below). Relations of the kind (7) should be seen as interface coupling conditions which supplement the equation (1),(4). They relate the traces of the restrictions $u^{l}:=\left.u\right|_{x<0}$ and $\left.u^{r}\right|_{x>0}$ of $u$ (the functions $u^{l, r}$ are assumed to be local solutions in the Kruzhkov sense of the corresponding conservation laws $u_{t}+f_{l, r}(u)=0$, in their respective domains of definition) and the corresponding flux values $F_{l, r}$ on the interface. This abstract formalism allows

\footnotetext{
${ }^{2}$ In contrast to boundary conditions, known to be a part of the definition of a differential operator and always made explicit in PDE literature, the choice of some particular interface coupling often remained a hidden assumption in the works devoted to discontinuous-flux conservation laws.

${ }^{3}$ We refer to [23] and references therein for some results on problems of the kind (5),(6) with general maximal monotone graphs $\beta$, including the delicate case of $x$-dependent BC graphs corresponding, e.g., to non-homogeneous and / or mixed boundary conditions.
} 
for conservative formulations $\left(F_{l}+F_{r}=0\right.$ is required, meaning that $u$ is a weak solution to (1),(4)) and more generally, it is suitable for problems with non-conservative coupling at the interface (see [17,22] for an example). By analogy with the classical terminology, let us call problems of the kind (1),(4),(2),(7) initial-interface value problems (IIVP, for short).

Let us briefly discuss the sense to be given to (7) in our setting of first-order conservation laws. The existence of traces of the solution and of the flux is ensured by the delicate "regularity" results of [54,61] that follow from the kinetic approach to conservation laws (cf. [63]) and from the technique of parametrized $H$-measures ( [59]). Due to this subtle regularity, (7) makes sense pointwise on the interface. Nonetheless, conditions (7) should not be considered literally. Indeed, in the case of scalar conservation law (1),(4) we have the functional dependence $F_{l}=f_{l}\left(u_{l}\right), F_{r}=-f_{r}\left(u_{r}\right)$, therefore the above ICC $(7)$ is over-determined unless $\mathcal{H}$ has a very particular shape. Therefore, if one wants to give sense to the problem (1),(4) supplied with an ICC (7) of general form, the condition (7) has to be relaxed. This occurs quite similarly to the way in which the Dirichlet boundary condition for a scalar conservation law is relaxed to yield the celebrated Bardos-LeRoux-Nédélec condition ( [26]). Similarly to what happens for the general initial-boundary value problems for conservation laws (see $\S 4.1$ and [21]), the relaxation can be interpreted as a projection procedure. Given a monotone graph $\mathcal{H} \subset \mathbb{R}^{2} \times \mathbb{R}^{2}$, one should consider the "closest" to $\mathcal{H}$ monotone graph $\widetilde{\mathcal{H}}$ satisfying the functional constraint

$$
\forall\left(u_{l}, u_{r} ; F_{l}, F_{r}\right) \in \tilde{\mathcal{H}}, \quad F_{l}=f_{l}\left(u_{l}\right), F_{r}=-f_{r}\left(u_{r}\right)
$$

(with, in addition, $F_{l}+F_{r}=0$ in the case of a conservative interface coupling). This means that in the definition of solutions to IIVP for discontinuous-flux conservation laws, the general formal ICC graph $\mathcal{H}$ in $(7)$ should be replaced with the projected effective $I C C$ graph $\widetilde{\mathcal{H}}$. The projection procedure, based on the use of Godunov numerical fluxes corresponding to nonlinearities $f_{l, r}$, is described in Section 4 below.

We expect that the IIVP of the form (1),(4),(2),(7) with the interpretation of (7) via the appropriate projection procedure enjoy well-posedness. Moreover, in some cases such problem can be efficiently approximated by a scheme that uses only the fluxes $f_{l, r}$ and the formal ICC graph $\mathcal{H}$. In this note, we do not prove for IIVP $(1),(4),(2),(7)$ precise results analogous to those of [21] for IBVP; this work is postponed for further research. Our goal is to provide some insight to the idea, illustrate it by examples, and advocate for the usefulness of the above interpretation in terms of interface coupling conditions of the whole variety (NU) of solvers for discontinuous-flux problems $(1),(4)$.

The outline of the paper is as follows. Section 1 recalls some of the known ideas and results originating from the work [15] of N.H. Risebro, K.H. Karlsen and the author. A basic convention on what is called "solution" is fixed in $\S 1.1$. The framework of $L^{1}$-dissipative germs and associated $\mathcal{G}$-entropy solutions is briefly recalled in $\S 1.2$. We devote a special attention to the multi-dimensional problem (1) (actually, the same tools are needed for the one-dimensional case with coupling condition which varies along the interface) in $\S 1.3$ and Appendix, where we collect key observations on important technical details needed for extension of the approach of [15]. Let us stress that the idea of such extension is, however, rather straightforward - except in what concerns construction of solutions and justification of existence, which may become a fairly delicate issue. In $\S 1.4$, main aspects of application of the germ approach and some of its shortcomings are summarized, then two recent developments and a new one are briefly described. These developments are detailed in Section 2, Section 3 and finally in Section 4 that is devoted to interface coupling conditions. Details for Section 2 can be found in the work [19] of D. Mitrović and the author; for Section 3, we refer to the work [11] (see also [8, 10]) of C. Cancès and the author. Section 4, which idea was already outlined in this introduction, transposes the tools of the works [20,21] of K. Sbihi and the author (recalled in $\S 4.1$ ) from the case of boundary-value problems to the case of interface coupling problems. The idea of such analogy originates from the [50] of C. Imbert and R. Monneau on Hamilton-Jacobi equations with discontinuous hamiltonians on networks. 


\section{Preliminaries: APPROACH By $L^{1}$-Dissipative GermS}

\subsection{On different notions of solution and admissibility conditions}

Let us make precise the following convention. In the sequel, considering conservation laws (1) with piecewise regular in $(t, x)$ flux with jump discontinuities arranged along a locally finite number of sufficiently regular interfaces (the union of interfaces being denoted by $\Sigma$ ), we will call "solution" any weak solution of the problem satisfying the Kruzhkov entropy inequalities locally in each subdomain of regularity of the flux $(t, x) \mapsto \mathfrak{f}(t, x ; \cdot)$. In some parts of the paper, conservativity at interfaces (i.e., the requirement that $u$ be a weak solution of (1)) will be relaxed.

Throughout this note, we will be interested in different kinds of admissibility conditions that should be imposed on "solutions", in the above sense, in order to achieve well-posedness results. These conditions will be formulated in various ways.

\subsection{Germs and $\mathcal{G}$-entropy solutions for the model problem(s) (1),(4)}

Our starting point is the theory developed in [15] in order to encompass all different $L^{1}$-contractive semigroups of solution (in the sense of $\S 1.1$ ) for the model discontinuous-flux conservation law (1),(4). In [15], these semigroups were put in one-to-one correspondence with subsets of $\mathbb{R} \times \mathbb{R}$ called $L^{1}$-dissipative germs. Roughly speaking, a germ $\mathcal{G}$ prescribes explicitly the possible jumps in $u$ at the interface $\{x=0\}$, in particular, one has $\left(u_{l}, u_{r}\right) \in \mathcal{G}$ if $u_{l} \mathbb{1}_{x<0}+u_{r} \mathbb{1}_{x>0}$ is an admissible solution in a neighbourhood of the interface $\{x=0\}$. Moreover, in practice one tends either to describe all possible solutions of this form (which leads to the notion of maximal germs), or to fix a smallest possible collection of such solutions which fully determines the admissibility of all other solutions (this leads to the notion of definite germs). Let us recall these definitions (see [15] for details and motivations).

Definition 1.1. A subset $\mathcal{G}$ of $\mathbb{R} \times \mathbb{R}$ is called $L^{1}$-dissipative germ if for all $\left(u_{l}, u_{r}\right) \in \mathcal{G}$ there holds, first ${ }^{4}$ $f_{l}\left(u_{l}\right)=f_{r}\left(u_{r}\right)$; and second, $q_{l, r}(\cdot, k)$ being the Kruzhkov entropy flux $\operatorname{sign}(\cdot-k)\left(f_{l, r}(\cdot)-f_{l, r}(k)\right)$,

$$
\forall\left(u_{l}, u_{r}\right),\left(v_{l}, v_{r}\right) \in \mathcal{G} \quad q_{l}\left(u_{l}, v_{l}\right)-q_{r}\left(u_{r}, v_{r}\right) \geq 0 .
$$

We say that $\mathcal{G}$ is maximal, if it is not a strict subset of some other $L^{1}$-dissipative germ. We say that $\mathcal{G}$ is definite, if there exists a unique maximal $L^{1}$-dissipative germ containing $\mathcal{G}$. Such unique maximal extension of $\mathcal{G}$ is denoted $\mathcal{G}^{*}$ in this case. We say that $\mathcal{G}^{*}$ is complete, if for every Riemann datum $u_{0}(x)=u_{l} \mathbb{1}_{x<0}+u_{r} \mathbb{1}_{x>0}$, $u_{l, r} \in \mathbb{R}$, there exists a solution in the sense of $\S 1.1$ which is self-similar (i.e., $u(t, x)=U(x / t)$ ) and such that $\left(U\left(0^{-}\right), U\left(0^{+}\right)\right) \in \mathcal{G}^{*}$.

Definition 1.2. Let $\mathcal{G}$ be a definite $L^{1}$-dissipative germ. A function $u \in L^{\infty}\left(\mathbb{R}_{+} \times \mathbb{R}\right) \cap C\left(\mathbb{R}_{+} ; L_{\text {loc }}^{1}(\mathbb{R})\right)$ is called $\mathcal{G}$-entropy solution of equation (1) with flux (4) if it is a solution in the sense of $\S 1.1$ and in addition it satisfies the following admissibility condition ${ }^{5}$ :

traces $u_{l}(\cdot)$ (from the left) and $u_{r}(\cdot)$ (from the right) of $u(\cdot, \cdot)$ on the interface $\{x=0\}$ fulfill the relation $\left(u_{l}(t), u_{r}(t)\right) \in \mathcal{G}^{*}$, for a.e. $t>0$.

\footnotetext{
${ }^{4}$ Later, non-conservative germs were considered, see in particular [22]. In this case, the conservativity condition $f_{l}\left(u_{l}\right)=f_{r}\left(u_{r}\right)$ should be omitted.

${ }^{5}$ The traces $u_{l, r}$ exist in the case of genuinely nonlinear fluxes $f_{l, r}$ because $u$ is a Kruzhkov entropy solution in domain $\{x \neq 0\}$ (see [61]). In general, the definition is a bit more involved because Temple kind singular mappings $V_{l, r}$ should be used to guarantee existence of traces of $V_{l, r} u_{l, r}$ of $V_{l, r}(u)$. The germ $\mathcal{G}$ in (9) is then replaced by the corresponding reduced germ defined by $V \mathcal{G}:=\left\{\left(V_{l}\left(c_{l}\right), V_{r}\left(c_{r}\right)\right) \mid\left(c_{l}, c_{r}\right) \in \mathcal{G}\right\}$. For the sake of simplicity, we will assume throughout this note that traces $u_{l, r}$ exist, which corresponds to $V_{l, r}=$ Id within the framework of [15].
} 
The above definition readily leads to a uniqueness proof. In order to justify existence, one needs in addition the completeness of the maximal extension $\mathcal{G}^{*}$ of $\mathcal{G}$ and one exploits the adapted entropy inequalities (cf. [24,30]), shown to be equivalent to the admissibility criterion (9):

$$
\forall\left(k_{l}, k_{r}\right) \in \mathcal{G} \quad|u-k(x)|_{t}+q(x ; u, k(x))_{x} \leq 0 \quad \text { in } \mathcal{D}^{\prime}\left(\mathbb{R}_{+} \times \mathbb{R}\right),
$$

where $k(x):=k_{l} \mathbb{1}_{x<0}+k_{r} \mathbb{1}_{x>0}$, the function $\eta(x ; \cdot):=|\cdot-k(x)| \equiv\left|\cdot-k_{l}\right| \mathbb{1}_{x<0}+\left|\cdot-k_{r}\right| \mathbb{1}_{x>0}$ is called adapted entropy, and $q(x ; \cdot, k(x)):=q_{l}\left(\cdot, k_{l}\right) \mathbb{1}_{x<0}+q_{r}\left(\cdot, k_{r}\right) \mathbb{1}_{x>0}$ is the entropy flux associated to $\eta(x ; \cdot)$. These definitions lead to the following well-posedness result which is the main claim of the theory of [15].

Theorem 1.3. Given a definite $L^{1}$-dissipative germ $\mathcal{G}$ which maximal extension $\mathcal{G}^{*}$ is complete, for every initial datum in $L^{\infty}(\mathbb{R})$ there exists one and only one $\mathcal{G}$-entropy solution to the discontinuous-flux conservation law (1) with flux (4).

We refer to [15] (see also [6] for a succinct presentation) for precisions and details.

\subsection{Some tools for the multi-dimensional problem}

There are few works devoted to the multi-dimensional case of problem (1). Actually, similar difficulties arise in the one-dimensional case with curved interfaces, and even in the model case (1),(4) with the interface coupling governed by a variable in time family of germs.

Extension of the general theory of [15] is essentially straightforward in what concerns uniqueness of solutions: we refer in particular to [12] and [14] for two complementary examples. Let us denote the interface by $\Sigma$, and let $\sigma \in \Sigma$ be its generic point. The key tool for such extensions is to associate a solution to a family of germs $\mathcal{G}(\cdot)$ variable along the interface. Recall that germs are associated to some given fluxes $f_{l, r}$. Here, we mean that $\mathcal{G}(\sigma)$ is an $L^{1}$-dissipative germ associated with scalar functions $f_{l, r}(\sigma ; \cdot)$ which are the normal components (in the direction of some arbitrarily fixed unit normal vector to the interface $\Sigma$, for $f_{r}(\sigma ; \cdot)$; in the opposite direction, for $f_{l}(\sigma ; \cdot)$ ) of the vector-valued one-sided traces at $\sigma \in \Sigma$ of the flux function $\mathfrak{f}(t, x ; \cdot)$. In compliance with the result of Theorem 1.3, for a.e. (with respect to the natural Hausdorff measure on $\Sigma$ ) point $\sigma \in \Sigma$, $\mathcal{G}(\sigma)$ should be a definite $L^{1}$-dissipative germ such that $\mathcal{G}^{*}(\sigma)$ is complete (in practice, we will mainly think of maximal $L^{1}$-dissipative germs). On can define the admissibility either by prescribing possible trace values analogously to (9):

$$
\left(u_{l}(\sigma), u_{r}(\sigma)\right) \in \mathcal{G}^{*}(\sigma) \text { for a.e. } \sigma \in \Sigma
$$

where $u_{l, r}(\cdot)$ are the traces of $u$ on $\Sigma$ in the direction of the already fixed unit normal vector to $\Sigma$ (for $u_{r}$ ) and in the opposite direction $\left(\text { for } u_{l}\right)^{6}$.

Alternatively, one can define the admissibility of solutions using a suitably extended family of inequalities of the kind (10), see (12) below. As in the model one-dimensional case of $\S 1.2$, both formulations are usually needed to establish uniqueness and existence (or, more generally, convergence of approximate methods) for the corresponding solutions, called $\mathcal{G}(\cdot)$-entropy solutions.

Let us stress that the family of germs $(\mathcal{G}(\sigma))_{\sigma \in \Sigma}$ cannot be arbitrary. To make the presentation concise, let us limit our attention to the situation where one considers one-dimensional homogeneous flux (4) with straight interface $\Sigma=\{(t, 0) \mid t>0, x=0\}$ parametrized by $\sigma=t$, and the admissibility is governed by a family of germs $(\mathcal{G}(t))_{t>0}$. One encounters such situation, e.g., in the constrained road traffic model introduced in [41] and revisited in [12]. Some measurability condition should be required on the family $\mathcal{G}(t)$ in this case. Indeed, consider the setting of $[15, \S 4.5]$ : to be specific, let us take $f_{l, r}: \mathbb{R} \rightarrow \mathbb{R}$ bijective, with $f_{l}$ an increasing and $f_{r}$ a decreasing function. In this situation, every maximal $L^{1}$-dissipative germ is a singleton of the form $\mathcal{G}_{a}:=\left\{\left(a, f_{r}^{-1} \circ f_{l}(a)\right)\right\}$. A non-measurable choice of $t \mapsto a(t)$ would lead to a family of germs $\mathcal{G}(t)=\mathcal{G}_{a(t)}$ for which existence of solution fails, indeed, (11) cannot hold because $t \mapsto u_{l}(t)=u\left(t, 0^{-}\right)$must be measurable

\footnotetext{
${ }^{6}$ Let us observe in passing that existence of such interface traces for general solutions (in the sense of $\S 1.1$ ) for the case of heterogeneous fluxes is proved only under the genuine nonlinearity restriction on $\mathfrak{f}(t, x ; \cdot)$ in each subdomain of smoothness of the flux: let us refer to [5].
} 
while (11) leaves no other choice than $u_{l}(t)=a(t)$. So, existence of a sufficiently rich family of measurable in $(t, x)$ functions $u$ which traces $u_{l, r}$ on $\Sigma$ fulfill (11) is an implicit measurability requirement on the considered family of germs. Further, in formulations of kind (10), measurability restriction appears explicitly as follows. Given a family $(\mathcal{G}(t))_{t>0}$ of maximal $L^{1}$-dissipative germs, we require that

$$
\forall\left(k_{l}, k_{r}\right) \in \mathbb{R} \times \mathbb{R} \quad|u-k(x)|_{t}+q(x ; u, k(x))_{x} \leq R_{\mathcal{G}(t)}\left(\left(k_{l}, k_{r}\right)\right) \delta_{0}(x) \quad \text { in } \mathcal{D}^{\prime}\left(\mathbb{R}_{+} \times \mathbb{R}\right)
$$

hold (with the same notation as in (10)) where the right-hand side features a suitably defined remainder term $R_{\mathcal{G}(\cdot)}$ sitting on the interface $\Sigma=\{x=0\}$. Then in order that (12) make sense, for all fixed $k_{l, r}$ the map $t \mapsto R_{\mathcal{G}(t)}\left(\left(k_{l}, k_{r}\right)\right)$ has to be measurable with respect to the one-dimensional measure on the interface. Observe that the choice of $R_{\mathcal{G}(\cdot)}$ is strongly restricted. Indeed, in order that for solutions (in the sense of $\left.\S 1.1\right)$ of (1),(4) equivalence between (11) and (12) hold, one needs the following properties to hold for a.e. $t>0$ :

$$
\begin{gathered}
\forall\left(k_{l}, k_{r}\right) \in \mathcal{G}(t) \lim _{r \downarrow 0} \frac{1}{2 r} \int_{t-r}^{t+r} R_{\mathcal{G}(s)}\left(\left(k_{l}, k_{r}\right)\right) d s=0, \\
\forall\left(k_{l}, k_{r}\right) \in \mathbb{R} \times \mathbb{R} \forall\left(c_{l}, c_{r}\right) \in \mathcal{G}(t) q_{r}\left(k_{r}, c_{r}\right)-q_{l}\left(k_{l}, c_{l}\right) \leq R_{\mathcal{G}(t)}\left(\left(k_{l}, k_{r}\right)\right) .
\end{gathered}
$$

Roughly speaking, because $\mathcal{G}(t)$ is definite, (13) implies (11) via a localization argument; while (14) is needed in order that solutions verifying (11) satisfy (12) for all $\left(k_{l}, k_{r}\right) \in \mathbb{R} \times \mathbb{R}$. In [15] and in several subsequent works, for Lipschitz continuous fluxes $f_{l, r}$ the following choice was suggested:

$$
R_{\mathcal{G}}\left(\left(k_{l}, k_{r}\right)\right):=M \operatorname{dist}\left(\left(k_{l}, k_{r}\right), \mathcal{G}\right)
$$

Measurability of $t \mapsto \operatorname{dist}\left(\left(k_{l}, k_{r}\right), \mathcal{G}(t)\right)$ is however a delicate issue, because $t \mapsto \mathcal{G}(t)$ is a set-valued map, moreover, the geometry of $\mathcal{G}(t)$ may undergo instantaneous changes under continuous change of $t$. We refer to Appendix for a further discussion of this technical issue, leading to a more involved that (15) choice of the remainder terms $R_{\mathcal{G}(\cdot)}\left(\left(k_{l}, k_{r}\right)\right)$ in adapted entropy inequalities (12).

Let us describe two applications. In [12], we developed a convergence proof for a finite volume scheme for approximation of solutions of the constrained road traffic model of [41]; it was based on formulation (12) with remainder terms $R_{\mathcal{G}(t)}$ given as in $(15)$, with $\mathcal{G}(\cdot)=\mathcal{G}_{\mathrm{F}(\cdot)}$ computed from the time-dependent constraint $\mathrm{F}(\cdot)$. The measurability of $t \mapsto R_{\mathcal{G}(\cdot)}\left(\left(k_{l}, k_{r}\right)\right)$ was evident in view of the explicit description of $\mathcal{G}_{\mathrm{F}(\cdot)}$ and the measurability of $F(\cdot)$. In [14] existence and uniqueness of vanishing viscosity admissible solutions was proved in a multi-dimensional setting, with only one sufficiently regular interface $\Sigma$. Let us stress that the proof used only the characterization (11), where the family of germs underlying the admissibility criterion was derived from the model case (4) studied in $\S 2.1^{7}$. Therefore the issue of defining and proving measurability of this family of germs was not addressed; heuristically, one can say that the fact that it should be seen as measurable is actually contained in the existence result. However, the proof of [14, Prop. 9] of equivalence of the admissibility conditions (11) and (the suitably adapted version of) (12) for solutions of (1) in the sense of $\S 1.1$ is not fully precise: indeed, the measurability of $t \mapsto \mathcal{R}_{G(t)}\left(\left(k_{l}, k_{r}\right)\right)$ was not justified. For this reason, in Appendix we provide a tentative definition of measurability of a general parametrized family of germs, and state that this notion is applicable to the problem with which [14, Prop. 9] is concerned.

\subsection{Towards more convenient admissibility criteria}

In $\oint 1.2, \oint 1.3$, admissibility conditions for solutions of (1) in the sense of $\S 1.1$ were expressed under the two equivalent forms:

\footnotetext{
${ }^{7}$ To be specific, the family $(\mathcal{G}(\sigma))_{\sigma \in \Sigma}$ consists of the vanishing viscosity germs $\mathcal{G}_{V V}$ of one-dimensional model cases (4) (see $\S 2.1$ ) associated with fluxes $f_{l, r}(\sigma, \cdot)$ where scalar $\sigma$-dependent one-dimensional fluxes $f_{l, r}$ are the normal components, at every point $\sigma \in \Sigma$, of the vector-valued fluxes on each side from $\Sigma$. We refer to [14] for the precise formulation.
} 
1. the explicit requirement that the couples $\left(u_{l}, u_{r}\right)$ of one-sided traces of an admissible solution belong to a specific subset $\mathcal{G}$ of $\mathbb{R} \times \mathbb{R}$, called $L^{1}$-dissipative germ;

2. the requirement that an admissible solution satisfies some global (up-to-the-interfaces) entropy inequalities, adapted from (3). In these inequalities one replaces the constants $k$ by more general (namely, piecewise constant) functions $k($.$) . As explained in \S 1.3$, one may need to incorporate into the adapted entropy inequalities some singular $k($.$) -dependent source terms.$

Equivalence means that the adapted entropy inequalities of $\mathbf{2}$. implicitly prescribe the restriction $\mathbf{1}$. on traces of solutions; and vice versa, solutions with traces prescribed by $\mathbf{1}$. automatically fulfill the adapted entropy inequalities of 2. Roughly speaking, formulations of kind 1. are most appropriate for uniqueness proofs; those of kind 2. seem better suited for existence analysis and study of convergence of various kinds of approximations. Yet in some situations we are aware of (see, e.g., [14]), formulation 1. should be used both for uniqueness and convergence analysis; in these situations, the existence analysis relies on quite indirect arguments, where the maximality of the underlying germ $\mathcal{G}$ and explicit knowledge of a sufficiently rich family of "obvious" solutions (e.g., obvious limits for the approximation procedure used in the construction) is required.

Both formulations 1. and 2. have considerable drawbacks. In general, serious work is needed in order to identify the germ $\mathcal{G}$ in $\mathbf{1}$. that encodes specific modeling assumptions at interfaces. Designing conditions of kind 2. may require less work, because the knowledge of a definite subset of a germ $\mathcal{G}$ (which is sometimes fairly small, see, e.g., $[9,30])$ is enough to write down the adapted entropy inequalities. However, the latter advantage disappears if the family of germs should vary along the interface. In this relation, it would be very interesting to develop tools in order to derive from modelling assumptions formulations 2. of the kind (12) (with some natural remainder terms), because they may not require the explicit knowledge of the underlying germ. At the present stage, only artificial formulations with $R_{\mathcal{G}}\left(\left(k_{l}, k_{r}\right)\right)=\operatorname{const} \operatorname{dist}\left(\left(k_{l}, k_{r}\right), \mathcal{G}\right)$ (or even worse, see $(56)$ in Appendix) are available for the general case.

But for the case of vanishing viscosity solutions (see $\S 2.1$ ), a much more natural entropy formulation (17) based only on Kruzhkov entropies like (3) with a remainder term (i.e., a formulation of the kind 2.) is appropriate: it works under an additional and rather strong restriction on the geometry of the fluxes (see (18)). In Section 2 we discuss the possibility of replacing (17) by singular Kruzhkov entropy inequalities which are easy to interpret and to discretize numerically, and which do not require condition (18) in order to be applicable. We call these new inequalities singular because they are not stable with respect to convergence of solutions in the natural for first-order conservation laws $L_{l o c}^{1}$-topology. For this reason, it turns out that these intuitively appealing inequalities are quite difficult to exploit in rigorous well-posedness analysis: to be specific, both existence and uniqueness proof are based on the identification of the underlying germ (approach 1.).

Next, in Section 4 we propose a variant of condition 1. where $\mathcal{G}$ is not given explicitly, but deduced from a specific coupling condition determined by a transmission map. A transmission map $\gamma$ tends to prescribe the value of $u_{r}$ as a monotone (possibly multivalued) function ${ }^{8}$ of $u_{l}: u_{r} \in \gamma\left(u_{l}\right)$. Many well-studied situations enter the framework of transmission maps (e.g.,the vanishing capillarity solutions for Buckley-Leverett equation, see $\S 3.2 .2$ and the non-conservative one-dimensional fluid-particle interaction model of [55], see $\S 3.2 .3$ ). As a result, new simple finite volume schemes for these models are derived, see $\S 3.3$.

Finally, in Section 4 we show that formulation of kind 1. can be implicitly prescribed in terms of interface coupling conditions (ICC) of the kind (7). The transmission map approach is easily reformulated in terms of ICC. We provide some facts of interest concerning interface coupling conditions in general situations with conservative and non-conservative coupling of conservation laws across an interface, and three examples.

\footnotetext{
${ }^{8}$ In general, $\gamma$ is a maximal monotone graph in $\mathbb{R} \times \mathbb{R}$ and " $u_{r}=\gamma\left(u_{l}\right)$ " $\operatorname{reads}\left(u_{l}, u_{r}\right) \in \gamma$.
} 


\section{Singular KruZhKov ENTROPy inEQUalities FOR CHARACTERIZATION OF VANISHING VISCOSITY LIMITS}

A number of works was devoted to studying limits, as $\varepsilon \rightarrow 0$, of solutions $u^{\varepsilon}$ of regularized equation (1):

$$
u_{t}^{\varepsilon}+\operatorname{div} \mathfrak{f}\left(t, x ; u^{\varepsilon}\right)=\varepsilon \Delta u^{\varepsilon} .
$$

This is the classical vanishing viscosity approach; for Lipschitz continuous fluxes $\mathfrak{f}$, it leads to the Kruzhkov notion of admissibility for equation (1).

\subsection{Vanishing viscosity limits for discontinuous-flux conservation laws}

In the discontinuous-flux setting, this approach boils down to fixing one specific notion of admissibility. This notion of admissibility can be described intrinsically, i.e., without appealing to the approximating sequence $u^{\varepsilon}$ for a given vanishing viscosity limit $u$. To our knowledge, for the model one-dimensional case with flux (4) two characterizations were firmly established:

(VV.a) the characterization by an Oleinik-like admissibility condition due to Diehl, see [44] (this approach generalizes the " $\Gamma$-condition" developed by the same author and the early "minimal jump condition" of [46]);

(VV.b) the characterization as $\mathcal{G}_{V V}$-entropy solutions, with an explicit description of the associated "vanishing viscosity germ" $\mathcal{G}_{V V}$, see $[14,15]$.

Further, in many practical cases (including the fluxes of the form $\mathfrak{f}(t, x ; u)=k(x) f(u)$ with piecewise constant $k$ ) one can rely upon

(VV.c) the characterization by a natural (though rough) Kruzhkov-like inequalities (17), first proposed in [66] (this admissibility condition appeared in a number of works including [2, 25, 64], the strongest results being proved by Karlsen, Risebro and Towers in [51]).

For the case (1),(4), the entropy inequalities of approach (VV.c) read:

$$
\forall k \in \mathbb{R}|u-k|_{t}+\left(\operatorname{sign}(u-k)(\mathfrak{f}(x ; u)-\mathfrak{f}(x ; k))_{x} \leq\left|f_{r}(k)-f_{l}(k)\right| \delta_{0}(x)\right.
$$

where $\delta_{0}$ is the Dirac measure concentrated at the origin. The proof of the fact that vanishing viscosity limits fulfill (17) is rather simple and it is very natural.

Concerning uniqueness, it is proved in [51] that under the crossing condition

$$
f_{r}(u)-f_{l}(u)<0<f_{r}(v)-f_{l}(v) \Rightarrow u<v
$$

inequalities (17) select, for a given initial datum $u_{0} \in L^{\infty}(\mathbb{R})$, at most one weak solution to (1),(4). We see that this weak solution is the unique vanishing viscosity limit associated with $u_{0}$ (it exists whenever the vanishing viscosity approximations converge). However, it is proved in [15] that whenever (18) fails, some Riemann problems for (1),(4) have non-unique solutions satisfying (17).

Our goal is to demonstrate that one can strengthen inequalities (17) so that they become able to characterize the vanishing viscosity limits also when the crossing condition (18) fails.

\subsection{Singular values and singular Kruzhkov entropy inequalities}

In the unpublished work [58], D. Mitrović proposed a new set of entropy inequalities for characterization of vanishing viscosity limits. In the model case (1),(4), it reads as follows:

$$
\exists p_{u}(\cdot) \quad \forall k \in \mathbb{R}|u-k|_{t}+(\operatorname{sign}(u-k)(\mathfrak{f}(x ; u)-\mathfrak{f}(x ; k)))_{x} \leq-\operatorname{sign}\left(p_{u}(t)-k\right)\left(f_{r}(k)-f_{l}(k)\right) \delta_{0}(x) .
$$


Here, $p_{u}(\cdot)$ can be seen as the value on the interface $\{x=0\}$ of some specific everywhere defined Borel representative of the solution $u$. The values of $p_{u}$ are not observable in practice, therefore we call them "singular values". Accordingly, we will say that (19) are singular Kruzhkov entropy inequalities. Inequalities (19) make sense and they represent the required strengthening of inequalities (17).

Indeed, well-posedness of (1),(4),(2) under the admissibility condition (19) is a particular case of the result of [19]. Uniqueness of solutions satisfying singular Kruzhkov entropy inequalities can be obtained by a painstaking case study. Both uniqueness and existence (via convergence of vanishing viscosity method) can be proved indirectly, with the help of the germ approach of $\S 1.2$. Roughly speaking, the principle of the existence proof in [19] is the same as in [14], up to serious technical difficulties relevant for the setting of $\S 2.3$ below.

Unfortunately, it seems that a direct proof of convergence of vanishing viscosity approximations $u^{\varepsilon}$ to a solution $u$ of (1),(4) satisfying (19) is compromised. Indeed, it is easy to obtain the analogue of (19) for solutions $u^{\varepsilon}$ of $(16),(4)$ : it is enough to take $p_{u^{\varepsilon}}(t):=u^{\varepsilon}(t, 0)$. However, we lack arguments showing strong compactness of $\left(p^{\varepsilon}(\cdot)\right)_{\varepsilon}$, which precludes us from using simple arguments of dominated convergence.

To sum up, in [19] we have established another intrinsic characterization of vanishing viscosity limits which, in contrast to (VV.c), is applicable to fluxes which crossing is not restricted:

(VV.c' ${ }^{\prime}$ the characterization by singular Kruzhkov entropy inequalities of the kind (19), see [19] (see also [42]). To conclude, let us observe that approach $\left(\mathbf{V V} \cdot \mathbf{c}^{\prime}\right)$ is based upon the fact that heuristically, the vanishing viscosity limit solutions are continuous - up to an interface layer - across the interface. The same idea of suitably relaxed continuity across the interface leads to a fourth characterization, which will be added to this list in $\S 3.2 .1$ :

(VV.d) the characterization of admissibility in terms of the transmission map $\gamma=\operatorname{Id}:=\{(u, u) \mid u \in \mathbb{R}\}$, see [11] and the next section.

\subsection{Existence for the multi-dimensional case: adapted viscosities}

Under a multi-dimensional analogue of the crossing condition (18), uniqueness of solutions to the Cauchy problem for (1) with piecewise regular fluxes satisfying the analogue of admissibility condition (17) is well known (see [51]). Recently, two works advocated that singular Kruzhkov entropy inequalities, which take the form

$\forall k \in \mathbb{R} \quad|u-k|_{t}+\operatorname{div}(\operatorname{sign}(u-k)(\mathfrak{f}(t, x ; u)-\mathfrak{f}(t, x ; k))) \leq-\operatorname{sign}(u-k)(\operatorname{div} \mathfrak{f}(t, x ; k))^{a c}-\operatorname{sign}\left(p_{u}-k\right)(\operatorname{div} \mathfrak{f}(t, x ; k))^{s}$

(in the sense of distributions) yield an admissibility criterion for (1) in the multidimensional case with inhomogenous fluxes, without any crossing restrictions on the fluxes. Here,

$$
\operatorname{div} \mathfrak{f}(t, x ; k)=(\operatorname{div} \mathfrak{f}(t, x ; k))^{a c}+(\operatorname{div} \mathfrak{f}(t, x ; k))^{s}
$$

is the Jordan decomposition of the Radon measure $\operatorname{div} \mathfrak{f}(t, x ; k)$ into its absolutely continuous part and its singular part.

Observe in passing that the difficulties discussed in $\S 1.3$ and in Appendix are not present in formulations (19),(20): indeed, measurability of $p_{u}$ is a part of the definition.

In [42], it was proved that $B V$ solutions satisfying inequalities of the kind (20) are unique for a quite general flux function $\mathfrak{f}$ with $S B V$ dependence in $(t, x), S B V$ being the special class of functions of bounded variation lacking the Cantor part. Existence of such solution is an open question. Less general piecewise Lipschitz functions $\mathfrak{f}$ with Lipschitz continuous jump manifolds were considered in [19]; but also less regular (merely $L^{\infty}$ ) solutions were studied. In the latter framework, both uniqueness and existence of solutions was proved. The proof of uniqueness does not truly differ from the model case; it is because uniqueness arguments support localization based, roughly speaking, on a local rectification of the interfaces. At the same time, our existence arguments require a delicate modification of the basic vanishing viscosity approximation. Roughly speaking, we first rectify the interfaces either with local Lipschitz changes of variables (using a partition of unity), or 
by a global change of variables which must have singularities (e.g., at the lower-dimensional manifolds where different pieces of interfaces intersect). Then the isotropic homogeneous viscosity in transformed coordinates is pulled back to the original coordinates; this yields a specific adapted viscosity operator. What we show in [19] is that, under rather non-restrictive assumptions on the interfaces (several examples are given) and under the genuine nonlinearity assumption on the fluxes $\mathfrak{f}(t, x ; \cdot)$, the resulting approximate solutions exist and converge to a solution of (1) admissible in the sense of the singular Kruzhkov entropy inequalities (20).

\section{TRAnsmission maPs AND hybridized Finite Volume schemes}

Let us present a construction that provides an implicit description of a particular family of germs. As the examples of $\S 3.2$ shows, this description is sometimes readily obtained from basic modeling considerations.

\subsection{Formalism of transmission maps and associated well-posedness results}

Given nonlinearities $f_{l, r}(\cdot)$ in problem $(1),(4)$ we denote by $\operatorname{God}_{l, r}(\cdot, \cdot)$ the corresponding Godunov numerical fluxes. Recall that the Godunov fluxes are defined by

$$
\operatorname{God}_{l, r}(a, b):= \begin{cases}\min _{u \in[a, b]} f_{l, r}(u) \quad, \quad a \leq b \\ \max _{u \in[b, a]} f_{l, r}(u) \quad, \quad a \geq b\end{cases}
$$

and in particular, they are non-decreasing in the first argument and non-increasing in the second argument.

Now, given a maximal monotone graph $\gamma$ in $\mathbb{R} \times \mathbb{R}$ (called transmission map in the sequel), consider the following pair of Cauchy-Dirichlet problems

$$
\left\{\begin{array} { l l } 
{ u _ { t } + f _ { l } ( u ) _ { x } = 0 } & { \text { in the domain } \{ t > 0 , x < 0 \} , } \\
{ u _ { | _ { t = 0 } } = u _ { 0 } } & { \text { for } x < 0 , } \\
{ u _ { | _ { x = 0 ^ { - } } } = \overline { u } _ { l } } & { \text { for } t > 0 , }
\end{array} \quad \left\{\begin{array}{ll}
u_{t}+f_{r}(u)_{x}=0 & \text { in the domain }\{t>0, x>0\} \\
u_{\left.\right|_{t=0}}=u_{0} & \text { for } x>0, \\
u_{\left.\right|_{x=0^{+}}}=\bar{u}_{r} & \text { for } t>0,
\end{array}\right.\right.
$$

in addition, denoting by $u_{l}(\cdot), u_{r}(\cdot)$ the left and right traces of $u(\cdot, \cdot)$ at $\{x=0\}$, we require the conservativity relation (later relaxed, see $\S 3.2 .3$ below)

$$
\text { for a.e. } t>0 f_{l}\left(u_{l}(t)\right)=f_{r}\left(u_{r}(t)\right) \text {. }
$$

Here measurable functions $\bar{u}_{l, r}(\cdot)$ stand for additional unknowns of the problem subject to the transmission condition encoded by $\gamma$ :

$$
\text { for a.e. } t>0\left(\bar{u}_{l}(t), \bar{u}_{r}(t)\right) \in \gamma \text {. }
$$

Solving (22),(23),(24) (i.e., finding $u$ and the two auxiliary unknown functions $\bar{u}_{l, r}$ ) is a way to produce solutions to (1),(4),(2) in the sense postulated in $\S 1$.1. It is shown in [11] that each choice of $\gamma$ corresponds to a choice

of a notion of admissibility, and that the resulting problem is well posed under some additional assumptions on $f_{l, r}$ (which amount to the completeness of the germ $\mathcal{G}[\gamma]$ defined below). Let us briefly explain the results of [11].

In order to analyze the admissibility notion encoded in $(22),(23),(24)$, let us first recall that in general, the left and right "effective traces" $u_{l, r}(\cdot)$ of $u(\cdot, \cdot)$ at $\{x=0\}$ differ from the prescribed left and right "desired traces" $\bar{u}_{l, r}(\cdot)$. Indeed, it is well known that the Dirichlet problem is over-determined (see [26] and $\S 4.1$ below). In the definition of solution to the Cauchy-Dirichlet problems (22), we use the standard Bardos-LeRoux-Nédélec relaxation procedure. Thus, the unknown desired traces $\bar{u}_{l, r}$ can be eliminated as functions of the effective traces $u_{l, r}$. To do this, recall that according to the Bardos-LeRoux-Nédélec procedure,

$$
\begin{aligned}
& \text { for a.e. } t>0 \quad u_{l, r}(t) \in I_{l, r}\left(\bar{u}_{l, r}(t)\right) \text {, where } \\
& I_{l, r}\left(u^{D}\right):=\left\{u \geq u^{D} \mid \forall k \in\left[u^{D}, u\right] f_{l, r}^{\nu}(k) \leq f_{l, r}^{\nu}(u)\right\} \bigcup\left\{u \leq u^{D} \mid \forall k \in\left[u, u^{D}\right] f_{l, r}^{\nu}(k) \geq f_{l, r}^{\nu}(u)\right\},
\end{aligned}
$$


$f_{l}^{\nu} \equiv f_{l}$ and $f_{r}^{\nu} \equiv-f_{r}$ being the normal (w.r.t. the interface) components of the fluxes in the respective domains $\{t>0, x<0\}$ and $\{t>0, x>0\}$. The definition (21) of Godunov flux helps to condense the above information:

$$
\text { (25) holds iff for a.e. } t>0 f_{l}\left(u_{l}(t)\right)=\operatorname{God}_{l}\left(u_{l}(t), \bar{u}_{l}(t)\right), \operatorname{God}_{r}\left(\bar{u}_{r}(t), u_{r}(t)\right)=f_{r}\left(u_{r}(t)\right)
$$

(the asymmetry in the expressions is due to the difference in the orientation of the exterior normal vector to the domains $\{t>0, x<0\}$ and $\{t>0, x>0\}$, i.e., to the fact that the definitions of $f_{l, r}^{\nu}$ in terms of $f_{l, r}$ differ the one from the other by the sign "-").

Now we are in a position to relate the transmission conditions (23),(24) to a specific $L^{1}$-dissipative germ. According to the above analysis, given a solution $u$ to (1),(4),(2) in the sense (22),(23),(24) (where the boundary condition is interpreted in the Bardos-LeRoux-Nédélec sense), the effective traces $u_{l, r}(\cdot)$ verify

$$
\text { for a.e. } t>0\left(u_{l}(t), u_{r}(t)\right) \in \mathcal{G}[\gamma]
$$

where

$$
\mathcal{G}[\gamma]:=\left\{\left(u_{l}, u_{r}\right) \mid \exists\left(\bar{u}_{l}, \bar{u}_{r}\right) \in \gamma f_{l}\left(u_{l}\right)=\operatorname{God}_{l}\left(u_{l}, \bar{u}_{l}\right)=\operatorname{God}_{r}\left(\bar{u}_{r}, u_{r}\right)=f_{r}\left(u_{r}\right)\right\} .
$$

The description (27),(28) contains both the flux transmission condition (23) and the state transmission condition (24) relaxed via the Bardos-LeRoux-Nédélec mechanism encoded by (26). This description fits the "germ" approach to conservation laws with discontinuous flux recalled in $\S 1.2$. Indeed, we have the following key observation (Prop. 2.8 in [11]):

\section{Given nonlinearities $f_{l, r}$ and a transmission map (a maximal monotone graph) $\gamma \in \mathbb{R} \times \mathbb{R}$, the subset $\mathcal{G}[\gamma]$ of $\mathbb{R}^{2}$ defined by (28) is an $L^{1}$-dissipative germ.}

This fact readily follows from the definition of $\mathcal{G}[\gamma]$ and the monotonicity of the Godunov fluxes $\operatorname{God}_{l, r}(\cdot, \cdot)$.

In addition, it is proved in [11] that in the case of unimodal ("bell-shaped") functions $f_{l, r}$ with compact support, $\mathcal{G}[\gamma]$ is a complete germ and therefore, it is a maximal $L^{1}$-dissipative germ. In particular, the model $(1),(4)$ associated with a transmission map $\gamma$ is well posed. Moreover, it is shown that every germ can be obtained from some well-chosen transmission map $\gamma$.

However, for fluxes of general (non-bell-shaped) form there may exist germs that are not of the form $\mathcal{G}[\gamma]$ for any transmission map $\gamma$, see $[11, \S 2.3]$.

\subsection{Examples. Extension to non-conservative coupling.}

Let us give three examples, the latter one requiring an extension of the transmission map approach to non-conservative coupling.

\subsubsection{Vanishing viscosity limits revisited: the map $\gamma=I d$}

To start with, we revisit the particular and important admissibility notion(s) discussed in $\S 2.1,2.2$. In fact, the simplest transmission map $\gamma=$ Id serves to describe the solutions of (1),(4) admissible in the sense of the classical vanishing viscosity criterion (and in the sense of the characterizations (VV.a), (VV.b), (VV.c' ${ }^{\prime}$ ), see $\S 2)$. The proof is given in [11].

The interest of this characterization by the identity transmission map (already mentioned above as (VV.d)) is that it fully reduces the understanding of vanishing viscosity limits to the continuity - up to an interface layer described in terms of Godunov fluxes - of solution $u$ across the interface. There is a simple link to the characterization (VV.c. ${ }^{\prime}$ ) described in $\S 2$ : namely, the value $\bar{u}_{l}(\cdot)=\bar{u}_{r}(\cdot)$ corresponding to a solution of $(22),(23),(24)$ with $\gamma=$ Id can be taken as the singular value $p_{u}(\cdot, 0)$ in the singular Kruzhkov entropy inequalities (19). This observation permits to stress the fact that, unfortunately, stability (with respect to the a.e. convergence) of admissible solutions in the sense $(22),(23),(24)$ is very difficult to prove directly, because convergence of the corresponding desired traces (三 singular values) cannot be established. Actually, 
we were unable to study these solutions without making appeal to the characterization (VV.b) with the germ $\mathcal{G}[\mathrm{Id}] \equiv \mathcal{G}_{V V}$

However, let us stress that the characterization (VV.d) enables for a particularly simple numerical approximation of vanishing viscosity limits, see $\S 3.3$. Actually, the idea of this numerical approximation appeared in the early work [40]. This approximation can also be applied in the multi-dimensional setting, even if the convergence analysis would be an involved task.

\subsubsection{Vanishing capillarity limits in the Buckley-Leverett context}

Continuity of $u^{\varepsilon}$ in more precise second-order models (cf. (16) above) is not necessarily relevant from physical grounds. The eloquent and important example is provided by one-dimensional hyperbolic Buckley-Leverett models considered as limits, as $\varepsilon \rightarrow 0$, of the vanishing capillarity models

$$
u_{t}^{\varepsilon}+f\left(x ; u^{\varepsilon}\right)_{x}=\varepsilon\left(\lambda\left(x ; u^{\varepsilon}\right) \nabla \pi\left(x ; u^{\varepsilon}\right)_{x}\right)_{x} .
$$

We refer to the works of Cancès et al. [33-37] for a description of solutions of the above models with $\varepsilon>0$.

The model case with flux (4) corresponds to a medium consisting of two rocks (on the left and on the right from the interface $\{x=0\}$ ) with distinct physical properties. In this case, the finer model (29) contains nonlinearities

$$
\lambda(x ; u)=\lambda_{l}(u) \mathbb{1}_{x<0}+\lambda_{r}(u) \mathbb{1}_{x>0}, \quad \pi(x ; u)=\pi_{l}(u) \mathbb{1}_{x<0}+\pi_{r}(u) \mathbb{1}_{x>0},
$$

with distinct $\lambda_{l, r}(\cdot)$ and $\pi_{l, r}(\cdot)$; the nonlinearities $\pi_{l, r}$ are capillary pressure profiles, and their shape strongly affects the behavior of $u^{\varepsilon}$ and of the limits, as $\varepsilon \rightarrow 0$, of $u^{\varepsilon}$.

Given $\pi_{l, r}(\cdot)$, the vanishing capillarity limits can be characterized intrinsically in terms of the associated germs (see [9]); but the transmission map approach provides a much more concise and easy-to-remember admissibility criterion. Namely, the weak formulation of approximate problems (29) means in particular that $\pi\left(\cdot ; u^{\varepsilon}(\cdot ; \cdot)\right)$ is continuous (in the sense of coinciding right and left traces) across the interface; physically, this corresponds to the continuity of the pressure, while the saturation undergoes an abrupt change on the border between the two rocks. Thus we have $\pi_{l}\left(u^{\varepsilon}\left(t, 0^{-}\right)\right)=\pi_{r}\left(u^{\varepsilon}\left(t, 0^{+}\right)\right)$for approximate solutions. This property is inherited at the limit, under the form $\pi_{l}\left(\bar{u}_{l}\right)=\pi_{r}\left(\bar{u}_{r}\right)$ where $\bar{u}_{l, r}$ are interpreted as the desired traces of the vanishing capillarity limit. Thus, the vanishing capillarity approximation corresponds to the transmission map $\gamma_{\pi_{l, r}}:=$ $\left\{\left(\pi_{l}^{-1}(p), \pi_{r}^{-1}(p) \mid p \in \mathbb{R}\right\}\right.$ (to be precise, $\pi_{l, r}(\cdot)$ are defined on $[0,1]$, and their inverses have to be extended to maximal monotone maps; we refer to [33,36] and [31] for details). It is proved in [10,11] that the vanishing capillarity limits corresponding to given $\pi_{l, r}(\cdot)$ are indeed characterized as the solutions of (22),(23),(24) with the associated transmission map $\gamma_{\pi_{l, r}}$.

To conclude the discussion of this example, let us mention that the multi-dimensional analogue of the problem considered here has a different nature. In this case, a hyperbolic equation for the saturation $u$ is coupled to an elliptic equation for velocity $v$. In this setting, no well-posedness theory is available because of the lack of regularity of the velocity field $v$; however, there is a natural adaptation of the numerical strategy based on transmission map, as exposed in $\S 3.3$ below. We refer to [8] for the description of the multi-dimensional model of flow in porous meduim consisting of two physically different rocks, for the associated IMPES kind finite volume scheme, and for numerical examples.

\subsubsection{The non-conservative case and the Burgers-particle model}

Assume that we are given a transmission map $\gamma$ and in addition, to every point of $\gamma$ we can associate a prescribed size of jump in the flux across the interface. In this case, instead of including the conservativity condition (23) into the definition of the germ, one has to fix a function $\psi$ defined on $\gamma \subset \mathbb{R}^{2}$ which prescribes the defect of conservation of $u$ at the interface. The germ (28) corresponds to $\psi \equiv 0$; given any dissipation map $\psi$ monotone w.r.t. the natural order on $\gamma$, i.e.,

$$
\psi: \gamma \mapsto \mathbb{R}, \quad \psi\left(\bar{u}_{l}, \bar{u}_{r}\right) \geq \psi\left(\underline{u}_{l}, \underline{u}_{r}\right) \text { whenever } \bar{u}_{l} \geq \underline{u}_{l} \text { and } \bar{u}_{r} \geq \underline{u}_{r}
$$


one can apply the same arguments as for the case $\psi \equiv 0$ and define

$$
\mathcal{G}[\gamma, \psi]:=\left\{\left(u_{l}, u_{r}\right) \mid \exists\left(\bar{u}_{l}, \bar{u}_{r}\right) \in \gamma \quad \begin{array}{l}
f_{l}\left(u_{l}\right)=\operatorname{God}_{l}\left(u_{l}, \bar{u}_{l}\right), \operatorname{God}_{r}\left(\bar{u}_{r}, u_{r}\right)=f_{r}\left(u_{r}\right), \\
\text { and } \operatorname{God}_{r}\left(\bar{u}_{r}, u_{r}\right)-\operatorname{God}_{l}\left(u_{l}, \bar{u}_{l}\right)+\psi\left(\bar{u}_{l}, \bar{u}_{r}\right)=0
\end{array}\right\} .
$$

As for the conservative case $\psi \equiv 0, \mathcal{G}[\gamma, \psi]$ is an $L^{1}$-dissipative germ (see $[11, \S 4.6]$ ).

Let us provide one example of such non-conservative coupling with a transmission map $\gamma$ and a dissipation map $\psi$. The following equation introduced in [55] models the resistance of a small particle located at $x=h(t)$ to motion of a one-dimensional fluid governed by the Burgers equation:

$$
u_{t}+\left(\frac{u^{2}}{2}\right)_{x}=-\lambda\left(u-h^{\prime}(t)\right) \delta_{0}(x-h(t))
$$

Here $\delta_{0}(\cdot)$ is the Dirac measure sitting at the origin, and the factor $-\lambda\left(u-h^{\prime}(t)\right)$ models the influence on the fluid of the particle located at $x=h(t)$ and moving with the relative (w.r.t. the fluid) velocity $\left(h^{\prime}(t)-u\right)$. Actually, in the coupled Burgers-particle model of [55], also the retroaction of the fluid on the particle is present; in this case, the particle evolution is governed by the drag force

$$
m h^{\prime \prime}(t)=\lambda\left(u(t, h(t))-h^{\prime}(t)\right)
$$

The meaning of $(31),(32)$ is not obvious because of the possible discontinuity of $u$ at $(t, h(t))$; the precise meaning, derived from regularization of is explained in [16,55]. A well-posedness theory of (31),(32) was constructed in $[16,17]$ on the basis of the "germ" formalism (the extension of germs to the non-conservative coupling is straightforward). The analysis of the interface coupling is essentially contained in the study of the motionless particle case $h \equiv 0$, see [22]. The $L^{1}$-dissipative (non-conservative) germ underlying the theory of (31) with $h \equiv 0$ is given by

$$
\mathcal{G}_{\lambda}=\left\{\left(u_{l}, u_{r}\right) \mid u_{l}-u_{r}=\lambda \text { or }\left(u_{r} \leq 0 \leq u_{l} \text { and }\left|u_{l}+u_{r}\right| \leq \lambda\right)\right\}
$$

Let us interpret the results of [22] in terms of transmission and dissipation maps. Given $\lambda>0$, we set

$$
\gamma_{\lambda}=\operatorname{Id}-\lambda:=\{(u, u-\lambda) \mid u \in \mathbb{R}\} \text { and } \psi_{\lambda}:\left(\bar{u}_{l}, \bar{u}_{r}\right) \mapsto \frac{\lambda}{2}\left(\bar{u}_{l}+\bar{u}_{r}\right)
$$

It is easy to check that the germ $\mathcal{G}_{\lambda}$ in (33) is precisely $\mathcal{G}\left[\gamma_{\lambda}, \psi_{\lambda}\right]$ obtained from $\gamma_{\lambda}, \psi_{\lambda}$ by procedure (30).

\subsection{Numerical approximation based on transmission maps}

Given an $L^{1}$-dissipative germ, construction of numerical approximations of $\mathcal{G}$-entropy solutions is delicate. There is a general method which is however quite heavy because it requires the a priori knowledge of completeness of $\mathcal{G}^{*}$ and the knowledge of the Riemann problem at the interface; then the Godunov numerical flux at the interface can be obtained and used to produce a global finite volume scheme (see [15, § 6.3] for details). In some examples we are aware of, much simpler convergent schemes can be designed (see [12,38] for an example of road traffic model and [16,22] for schemes developed for non-conservative example (31)).

In the particular case of germs $\mathcal{G}(\gamma)$ obtained from a given transmission maps $\gamma$, the knowledge of the Riemann solver is not necessary because the Godunov flux can be obtained by a simple calculation that involves the expressions of $\gamma$ and of left and right Godunov fluxes $\operatorname{God}_{l, r}$. Indeed, the transmission map $\gamma$ leads to the following expression of numerical flux at the interface:

$$
F_{\gamma}(a, b):=\operatorname{God}_{l}\left(a, \bar{u}_{l}\right)=\operatorname{God}_{r}\left(\bar{u}_{r}, b\right) \text { where }\left(\bar{u}_{l}, \bar{u}_{r}\right) \in \gamma \text { is such that } \operatorname{God}_{l}\left(a, \bar{u}_{l}\right)=\operatorname{God}_{r}\left(\bar{u}_{r}, b\right)
$$


While the point $\left(\bar{u}_{l}, \bar{u}_{r}\right) \in \gamma$ defining $F_{\gamma}(a, b)$ in (34) may not be unique, all the different choices give the same value of $F_{\gamma}(a, b)$. Existence of such points $\left(\bar{u}_{l}, \bar{u}_{r}\right) \in \gamma$ actually requires some $a$ priori conditions ${ }^{9}$ that ensure that the image of $p \mapsto \operatorname{God}_{r}\left(\bar{u}_{r}(p), b\right)-\operatorname{God}_{l}\left(a, \bar{u}_{l}(p)\right)$ contains zero; here,

$$
p \mapsto\left(\bar{u}_{l}(p), \bar{u}_{r}(p)\right) \in \gamma \text { is a monotone parametrization of } \gamma \text {. }
$$

In is easy to extend the definition of the fluxes for the non-conservative case: in this situation, the left flux $F_{\gamma, \psi}^{-}:=\operatorname{God}_{l}\left(a, \bar{u}_{l}\right)$ and the right flux $F_{\gamma, \psi}^{+}:=\operatorname{God}_{r}\left(\bar{u}_{r}, b\right)$ are deduced from resolution of the equation

$$
\operatorname{God}_{r}\left(\bar{u}_{r}(p), b\right)-\operatorname{God}_{l}\left(a, \bar{u}_{l}(p)\right)+\psi\left(\bar{u}_{l}(p), \bar{u}_{r}(p)\right)=0 \text { with parametrization (35). }
$$

The definition of numerical fluxes (34) requires solution of one nonlinear scalar equation per time step (because of this additional unknown, we say that the scheme is hybridized). In practice, numerical resolution of equations appearing in (34) or more generally, in (36) is quite fast using the regula falsi method (see [10] for a particular example). Based on the fluxes $\left.\operatorname{God}_{l, r}(\cdot, \cdot)\right)$ applied for $x<0$ and $x>0$ and on the new flux $F_{\gamma}(\cdot, \cdot)$ applied at $\{x=0\}$, it is easy to construct a finite volume scheme which is almost explicit (except for the above mentioned scalar equation to be solved per time step).

It is shown in [11] that $F_{\gamma}$ is exactly the Godunov numerical flux associated with the Riemann solver at the interface. More importantly, any other consistent with $f_{l, r}$ monotone numerical fluxes $R_{l, r}$ can be used in (34) in the place of $\operatorname{God}_{l, r}$; the resulting scheme for the discontinuous-flux problem (1),(4) subject to admissibility condition is monotone and convergent. We refer to [11] for numerical examples using in particular the Rusanov numerical fluxes for $R_{l, r}$.

Finally, let us point out that it is easy to obtain from the transmission map not only the Godunov flux at interface, but also the interface Riemann solver. To this end, denote by $\mathcal{R}_{l}(a, b), \mathcal{R}_{r}(a, b)$ the Riemann solvers for the fluxes $f_{l}, f_{r}$ respectively, where $a \mathbb{1}_{x<x_{0}}+b \mathbb{1}_{x>x_{0}}$ (with $x_{0}<0$ and $x_{0}>0$. respectively) are the Riemann data. Now, consider Riemann data corresponding to a jump at the interface point $x_{0}=0$. Then,

(1) given $a$ and $b$, one has to find $\bar{u}_{l, r}$ from (34) (or, more generally, from (36));

(2) with $a$ and $\bar{u}_{l}$ at hand, take in the domain $\{x<0\}$ the waves of negative speed given by $\mathcal{R}_{l}\left(a, \bar{u}_{l}\right)$;

(3) with $\bar{u}_{r}$ and $b$ at hand, take in the domain $\{x>0\}$ the waves of positive speed given by $\mathcal{R}_{r}\left(\bar{u}_{r}, b\right)$;

(4) juxtapose the so obtained solutions in the two subdomains separated by the interface $\{x=0\}$.

If the Riemann solvers are discretized by replacing rarefactions with sequences of small non-entropic shocks, it is enough to have in discretized rarefactions a shock of zero speed: one obtains a discretized Riemann solver for the interface. This permits, in particular, to apply the wave-front tracking construction for numerical approximation of solutions of $(22),(23),(24)$. To sum up, the wave-front tracking construction requires the knowledge of the Riemann solvers $\mathcal{R}_{l, r}$ and the resolution, at each time where a wave reaches the interface, of the scalar nonlinear equation in (34) or (36). At the same time, the analysis of such wave-front tracking algorithm is not straightforward because, first, $B V$ estimates may fail in the discontinuous-flux setting (see [1]); and second, the bound of the total number of fronts may prove to be delicate. In applications, singular mappings and Temple kind functionals may prove useful, see, e.g., [41].

\section{INTERFACE COUPLING CONDITIONS}

In this section, we introduce a rather natural formalism for describing possible coupling of Kruzhkov entropy solutions to (1),(4) in the subdomains $\{x>0\},\{x<0\}$ across the interface $\{x=0\}$. It is inspired by the

\footnotetext{
${ }^{9}$ It is obvious that some discontinuous-flux problems may not admit solutions for any data; the simplest example, in the conservative situation, is when the ranges of $f_{l}$ and $f_{r}$ are disjoint. While approximations (numerical ones, or by vanishing viscosity regulatization, etc.) can sometimes be defined, uniform bounds on these approximations may fail to exist; which leads to a lack of convergence of approximate solutions and explains non-existence of solutions for the limit problem.
} 
works [20,21] of K. Sbihi and the author, which setting is recalled in $\S 4.1$ below, and by the observations made by C. Imbert and R. Monneau in [50] in the related context of Hamilton-Jacobi equations on networks.

\subsection{Boundary conditions for scalar conservation laws revisited}

For simplicity, consider the IBVP for a scalar conservation law with flux $f$ such that $f^{\prime} \neq 0$ on intervals:

$$
\begin{aligned}
& u_{t}+f(u)_{x}=0 \text { for }(t, x) \in \mathbb{R}_{+}^{*} \times \mathbb{R}_{-}^{*}, \\
& \left.(u, f(u))\right|_{x=0^{-}} \in \beta \\
& \left.u\right|_{t=0}=u_{0}
\end{aligned}
$$

where $\beta$ is a maximal monotone graph in $\mathbb{R} \times \mathbb{R}$, and the trace of $u$ exists in the strong sense due to the results of [61]. The second line in (37) stands for a (formal) boundary condition. The classical Dirichlet condition $\left.u\right|_{x=0^{-}}=u^{D}$ corresponds to $\beta=\left\{u^{D}\right\} \times \mathbb{R}$. In the general case, one can consider $\beta$ depending on the point of the boundary (e.g., for the case where $u^{D}$ depends on $t$ ). Monotonicity of $\beta$, which can be expressed as

$$
\forall(u, F),(\hat{u}, \hat{F}) \in \beta \quad \operatorname{sign}(u-\hat{u})(F-\hat{F}) \geq 0,
$$

is a natural property to be required of a boundary condition of the form we consider. Indeed, it ensures $L^{1}$ contractivity of the solution semigroup (like for the problem in the whole space, as established by Kruzhkov [52]). In fact, assume that $u, \hat{u}$ are two solutions verifying

$$
u_{t}+\mathcal{F}[u]_{x}=0, \quad \hat{u}_{t}+\mathcal{F}[\hat{u}]_{x}=0
$$

where $\mathcal{F}[\cdot]$ represents some procedure to compute the flux from the solution (one has $\mathcal{F}=f(u)$ for problem (37); but one can also think of $\mathcal{F}=f(u)-\varepsilon u_{x}$ for its vanishing viscosity approximation). Assume that the respective traces of the solutions and the associated normal fluxes (denoted by operator $\gamma$ ) exist in a strong sense, and assume for a moment that the satisfy literally the interface conditions, i.e.,

$$
\text { for a.e. } t>0((\gamma u)(t),(\gamma \mathcal{F}[u])(t)),((\gamma \hat{u})(t),(\gamma \mathcal{F}[\hat{u}])(t)) \in \beta \text {. }
$$

From the local Kruzhkov entropy formulation of (37) one deduces that the local Kato inequalities hold:

$$
\forall \xi \in C_{0}^{\infty}\left(R_{+}^{*} \times \mathbb{R}_{-}^{*}\right)^{+} \quad-\int_{R_{+} \times \mathbb{R}_{-}}\left(|u-\hat{u}| \xi_{t}+\operatorname{sign}(u-\hat{u})(\mathcal{F}[u]-\mathcal{F}[\hat{u}]) \xi_{x}\right) \leq 0 .
$$

Then, we can take test function $\xi \in C_{0}^{\infty}\left(R_{+}^{*} \times \mathbb{R}\right)$ and approximate it with truncated test function $\xi \xi_{h}$, the truncation $\xi_{h}$ being zero in a neighbourhood of the boundary $\{x=0\}$ and converging to 1 a.e., as $h \rightarrow 0$. Due to existence of strong traces, one finds ${ }^{10}$ at the limit $h \rightarrow 0$ the up-to-the-boundary Kato inequality

$$
-\int_{R^{+} \times \mathbb{R}-}\left(|u-\hat{u}| \xi_{t}+\operatorname{sign}(u-\hat{u})(\mathcal{F}[u]-\mathcal{F}[\hat{u}]) \xi_{x}\right) \leq-\int_{R_{+}} \operatorname{sign}(\gamma u-\gamma \hat{u})(\gamma \mathcal{F}[u]-\gamma \mathcal{F}[\hat{u}]) \leq 0
$$

now with test functions $\xi \in C_{0}^{\infty}\left(R_{+}^{*} \times \mathbb{R}\right)^{+}$. The last inequality is due to (39) and to the monotonicity of $\beta$. Taking (by approximation) $\xi \equiv \mathbb{1}_{\left[t_{1}, t_{2}\right]}(t)$, we find that for $t_{1}<t_{2}$ there holds $\left\|u\left(t_{2}, \cdot\right)-\hat{u}\left(t_{2}, \cdot\right)\right\|_{L^{1}\left(\mathbb{R}_{-}\right)} \leq$ $\left\|u\left(t_{1}, \cdot\right)-\hat{u}\left(t_{1}, \cdot\right)\right\|_{L^{1}\left(\mathbb{R}_{-}\right)}$. This means that at least formally, the boundary condition of the form $\left.(u, f(u))\right|_{x=0^{-}} \in$ $\beta$ with monotone $\beta$ contributes to dissipate the $L^{1}$ distance between solutions of (37). In particular, monotonicity

\footnotetext{
${ }^{10}$ The computation is rigorous for $F[u]=f(u)$, because whenever $\gamma u=\gamma \hat{u}$, also $\gamma \mathcal{F}[u]=f(\gamma u) \equiv f(\gamma \hat{u})=\gamma \mathcal{F}[\hat{u}]$ holds true. More generally, e.g. for $F[u]=f(u)-\varepsilon u_{x}$, one has to consider the multi-valued function sign max $_{\text {in }}$ the right-hand side of (41) and to use finer arguments (cf. $[13,45]$ ) in order to deduce that the contribution of the boundary terms to the Kato inequality is dissipative.
} 
assumption on $\beta$ settles the question of uniqueness of solutions. Actually, all classical boundary conditions (Dirichlet, Neumann (zero-flux), Robin, obstacle) are of the maximal monotone kind.

Now, it is evident (and well known, at least for the Dirichlet BC case) that in general, existence of solutions to (37) (in the local Kruzhkov entropy sense, with boundary condition satisfied pointwise) is compromised. This is due to the fact that the couple $\left(u\left(t, 0^{-}\right), f\left(u\left(t, 0^{-}\right)\right)\right)$of solution and flux traces must belong to the graph of the flux function $f$; yet it may happen that the intersection of $\beta$ and $f$ is reduced to a point, or even that this intersection is empty. The relaxed formulation of the Dirichlet boundary condition proposed by Bardos, LeRoux, Nédélec [26] provided the following interpretation of the condition " $u\left(t, 0^{-}\right)=u^{D}$ " : at every point of the boundary, $u\left(t, 0^{-}\right.$) should belong to some set $I\left(u^{D}\right) \subset \mathbb{R}$ (made explicit in (25) of Section 3) which depends on $u^{D}$ and on the graph of $f(\cdot)$. This subset is in general much larger that the singleton $\left\{u^{D}\right\}$. The relevancy of the BLN interpretation is due to the fact that approximation of the Dirichlet problem by second-order equations (the vanishing viscosity approximation) produces limits that are precisely the Bardos-LeRoux-Nédélec solutions (see [26]). In order to generalize the approach and the result of [26], let us reformulate it as follows:

$$
\left.(u, f(u))\right|_{x=0^{-}} \in \tilde{\beta},
$$

where $\tilde{\beta}$ is the one among all maximal monotone subgraphs of $\{(z, f(z) \mid z \in \mathbb{R}\}$ that is the closest one to $\beta$. The precise meaning of "the closest one" and several characterizations of $\tilde{\beta}$ (given $f$ and $\beta$ ) are given in [21]. Actually, the operation $\beta \mapsto \tilde{\beta}$ is a projection ${ }^{11}$. Here, we focus on the characterization of $\tilde{\beta}$ in terms of the Godunov flux God: $\mathbb{R}^{2} \mapsto \mathbb{R}$ associated with $f$ :

$$
\tilde{\beta}=\{(u, f(u)) \mid \exists(\bar{u}, F) \in \beta \text { such that } F=f(u)=\operatorname{God}(u, \bar{u})\} .
$$

To briefly summarize the results of [21], let us say that given a maximal monotone graph $\beta$, for every $L^{\infty}$ initial datum, there exists a unique $L^{\infty}$ function $u$ which solves (37) in the sense that $u$ is a Kruzhkov entropy solution away from the boundary, and it fulfills the boundary condition in the projected sense (42),(43). Moreover, $u$ is the limit of approximations based on the vanishing viscosity regularization of problem $(37)^{12}$.

Let us stress that the monotonicity of $\beta$ implies the monotonicity of $\tilde{\beta}$, which is crucial for uniqueness. The maximality of $\beta$ implies the maximality of $\tilde{\beta}$, which is a sufficient condition for existence of solutions in the above sense; but $\beta$ need not be maximal in general.

\subsection{The framework of interface coupling conditions}

Now, let us suggest an analogous formulation for the model discontinuous-flux conservation law (1),(4). Throughout this section, $f_{l, r}$ are fixed continuous functions of $\mathbb{R}$. Assuming that all the trace values mentioned hereafter exist, we are intended to relate not just two values (the solution and the flux at the interface) as in $\S 4.1$ but the four values $u_{l}, u_{r}, F_{l}, F_{r}$, defined as functions of the interface point. As above, we limit our attention to homogeneous (not dependent on the point chosen on the interface) interface coupling conditions. Here $u_{l}(t)$ and $u_{r}(t)$ are the trace values of $u(t, \cdot)$ at $x=0^{-}$and $x=0^{+}$, respectively; while $F_{l}(t)$ and $F_{r}(t)$ are the traces at time $t$ of the normal outgoing fluxes on the boundaries of the subdomains $\{x<0\}$ and $\{x>0\}$, respectively. It is implicitly assumed, writing equation $(1),(4)$, that $u$ is a weak solution and therefore there holds the conservativity relation

$$
F_{l}+F_{r}=0 .
$$

\footnotetext{
${ }^{11}$ Therefore the result of [21] is typical of singular limit problems: the boundary condition, satisfied in the literal sense for the approximate vanishing viscosity problems with $\varepsilon>0$, is projected as $\varepsilon \rightarrow 0$ on the subset of all boundary conditions that can be possibly realized at the limit $\varepsilon=0$ : e.g., the BLN condition can be seen as the projection of the Dirichlet boundary condition.

${ }^{12}$ To be precise, one may need a preliminary approximation of $\beta$ by a sequence of rapidly growing graphs, in order to ensure uniform $L^{\infty}$ bounds on approximate solutions. This difficulty is not present for Dirichlet or obstacle conditions, but the use of such two-step approximation may be necessary, for instance, to give sense to the zero-flux boundary condition (see [20,21]).
} 
In the sequel, we will say that the situation (44) is a conservative interface coupling condition. In general we are sometimes interested in relaxing the requirement (44) (this is needed to study situations with non-conservative coupling as in $\S 3.2 .3)$. By analogy with the setting of $\S 4.1$, we will see the set of all points $\left(u_{l}, u_{r} ; F_{l}, F_{r}\right) \in \mathbb{R}^{4}$ allowed by a given ICC as a graph $\mathcal{H} \subset \mathbb{R}^{2} \times \mathbb{R}^{2}$.

The notion of monotonicity suitable for graphs in $\mathbb{R} \times \mathbb{R}$ cannot be directly copied here, because there is no total order relation on $\mathbb{R}^{2}$. Instead, motivated by the definition of $L^{1}$-dissipativity of germs, one could define the suitable notion of monotonicity of $\mathcal{H} \subset \mathbb{R}^{2} \times \mathbb{R}^{2}$ by the relation

$$
\forall\left(u_{l}, u_{r} ; F_{l}, F_{r}\right),\left(\hat{u}_{l}, \hat{u}_{r} ; \hat{F}_{l}, \hat{F}_{r}\right) \in \mathcal{H} \quad \operatorname{sign}\left(u_{l}-\hat{u}_{l}\right)\left(F_{l}-\hat{F}_{l}\right)+\operatorname{sign}\left(u_{r}-\hat{u}_{r}\right)\left(F_{r}-\hat{F}_{r}\right) \geq 0
$$

We will take a slightly less restrictive definition of monotonicity:

$$
\forall\left(u_{l}, u_{r} ; F_{l}, F_{r}\right),\left(\hat{u}_{l}, \hat{u}_{r} ; \hat{F}_{l}, \hat{F}_{r}\right) \in \mathcal{H} \quad\left[u_{l}-\hat{u}_{l}, F_{l}-\hat{F}_{l}\right]+\left[u_{r}-\hat{u}_{r}, F_{r}-\hat{F}_{r}\right] \geq 0
$$

where we have used the notation

$$
\text { for } a, b \in \mathbb{R} \quad[a, b]:=b(\operatorname{sign} a)+|b| \mathbb{1}_{\{0\}}(a),
$$

i.e., $[a, b]=b \operatorname{sign}(a)$ except in the case $a=0$, where we set $[a, b]=|b|$.

Definition 4.1 (1-monotonicity). We say that a subset $\mathcal{H}$ of $\mathbb{R}^{2} \times \mathbb{R}^{2}$ is 1 -monotone if inequalities (46) hold. If the strengthened inequalities (45) hold, we say that $\mathcal{H}$ is fully 1 -monotone.

Observe that the positivity of sums of the form $\left(u_{l}-\hat{u}_{l}\right)\left(F_{l}-\hat{F}_{l}\right)+\left(u_{r}-\hat{u}_{r}\right)\left(F_{r}-\hat{F}_{r}\right)$ would give rise to a different notion of monotonicity (we could call it 2-monotonicity). The 1-monotonicity introduced here is adapted to problems with underlying $L^{1}$-contraction property (witnessed via a Kato inequality): the argument is analogous to the one that led from (38) to (41) in $\S 4.1$.

In the sequel, let us denote by $\mathfrak{H}$ the set of all 1-monotone graphs in $\mathbb{R}^{2} \times \mathbb{R}^{2}$, and denote by $\mathfrak{H}_{0}$ the set of all conservative 1-monotone graphs in $\mathbb{R}^{2} \times \mathbb{R}^{2}$. Further, we will say that $\mathcal{H}$ is canonical if for all $\left(u_{l}, u_{r} ; F_{l}, F_{r}\right) \in \mathcal{H}$ there holds $F_{l}=f_{l}\left(u_{l}\right)$ and $F_{r}=-f_{r}\left(u_{r}\right)$. Accordingly, we will denote by $\widetilde{\mathfrak{H}}$ the set of all canonical 1-monotone graphs in $\mathbb{R}^{2} \times \mathbb{R}^{2}$; the notation $\widetilde{\mathfrak{H}}_{0}$ will be used for the subset of $\widetilde{\mathfrak{H}}$ consisting of conservative graphs.

Now, we are in a position to define the projection operator $\sim: \mathfrak{H} \rightarrow \widetilde{\mathfrak{H}}$ by

$$
\begin{array}{r}
\widetilde{\mathcal{H}}:=\left\{\left(u_{l}, u_{r} ; F_{l}, F_{r}\right) \in \mathbb{R}^{2} \times \mathbb{R}^{2} \mid \exists\left(\bar{u}_{l}, \bar{u}_{r} ; F_{l}, F_{r}\right) \in \mathcal{H}\right. \\
\left.F_{l}=f_{l}\left(u_{l}\right)=\operatorname{God}_{l}\left(u_{l}, \bar{u}_{l}\right),-F_{r}=f_{r}\left(u_{r}\right)=\operatorname{God}\left(\bar{u}_{r}, u_{r}\right)\right\}
\end{array}
$$

$\left(\operatorname{God}_{l, r}\right.$ being the Godunov fluxes associated with $f_{l, r}$, as given in (21)). Indeed, the projected ICC graph $\widetilde{H}$ is canonical by definition; in order to see that it is 1 -monotone (due to the 1 -monotonicity of $\mathcal{H}$ ), we prove the following:

Proposition 4.2. Assume $\mathcal{H}$ is 1-monotone. Then $\widetilde{H}$ defined by (48) is fully 1-monotone.

One can also observe that the projection of a conservative graph is conservative: $\mathcal{H} \in \mathfrak{H}_{0}$ implies $\widetilde{\mathcal{H}} \in \widetilde{\mathfrak{H}}_{0}$.

Proof of Proposition 4.2. The monotonicity of $\widetilde{\mathcal{H}}$ results from the monotonicity of the Godunov fluxes $G_{0} d_{l, r}$. The proof is by a straightforward case study. Given two points

$$
\left(u_{l}, u_{r} ; F_{l}, F_{r}\right),\left(\hat{u}_{l}, \hat{u}_{r} ; \hat{F}_{l}, \hat{F}_{r}\right) \in \widetilde{\mathcal{H}}
$$

we take the associated points

$$
\left(\bar{u}_{l}, \bar{u}_{r} ; F_{l}, F_{r}\right),\left(\overline{\hat{u}}_{l}, \overline{\hat{u}}_{r} ; \hat{F}_{l}, \hat{F}_{r}\right) \in \mathcal{H}
$$


(with the notation of (48)) and consider different cases of ordering of values $u_{l, r}, \hat{u}_{l, r}$ in relation with ordering of $\bar{u}_{l, r}, \overline{\hat{u}}_{l, r}$. Our claim is that in all cases,

$$
\left[u_{l, r}-\hat{u}_{l, r}, F_{l, r}-\hat{F}_{l, r}\right] \geq\left[\bar{u}_{l, r}-\overline{\hat{u}}_{l, r}, F_{l, r}-\hat{F}_{l, r}\right] .
$$

It is enough to look separately at the subscripts $l, r$; let us write "l" to shorten the notation.

We have the following collection of cases, which covers all logical possibilities.

- $\operatorname{sign}\left(u_{l}-\hat{u}_{l}\right)=\operatorname{sign}\left(\bar{u}_{l}-\overline{\hat{u}}_{l}\right) \neq 0$ : in this case, the two sides of the desired inequality (49) are identical.

- $\operatorname{sign}\left(u_{l}-\hat{u}_{l}\right)=-\operatorname{sign}\left(\bar{u}_{l}-\overline{\hat{u}}_{l}\right) \neq 0$ : in this case, there are two symmetric possibilities that are treated analogously. For instance, consider the situation where $u_{l}<\hat{u}_{l}$ but $\bar{u}_{l}>\overline{\hat{u}}_{l}$. Then by the definition of $\bar{u}_{l}, \overline{\hat{u}}_{l}$ and from the monotonicity of $\operatorname{God}_{l}(\cdot, \cdot)$ (non-decreasing in the first variable, non-increasing in the second one) we have

$$
F_{l}=\operatorname{God}_{l}\left(u_{l}, \bar{u}_{l}\right) \leq \operatorname{God}_{l}\left(\hat{u}_{l}, \overline{\hat{u}}_{l}\right)=\hat{F}_{l}
$$

Hence

$$
\begin{aligned}
{\left[u_{l}-\hat{u}_{l}, F_{l}-\hat{F}_{l}\right]=\operatorname{sign}\left(u_{l}-\hat{u}_{l}\right)\left(F_{l}-\hat{F}_{l}\right)=} & -\left(F_{l}-\hat{F}_{l}\right)=\left|F_{l}-\hat{F}_{l}\right| \\
& \geq \operatorname{sign}\left(\bar{u}_{l}-\overline{\hat{u}}_{l}\right)\left(F_{l}-\hat{F}_{l}\right)=\left[\bar{u}_{l}-\overline{\hat{u}}_{l}, F_{l}-\hat{F}_{l}\right] .
\end{aligned}
$$

- $u_{l}$ coincides with $\bar{u}_{l}$ or $\hat{u}_{l}$ coincides with $\overline{\hat{u}}_{l}$ : here, the result is achieved by a minor extension of the above arguments, keeping in mind the definition (47) of the bracket.

In conclusion, we have justified (49); now the 1-monotonicity of $\widetilde{\mathcal{H}}$ is an immediate consequence of the 1monotonicity of $\mathcal{H}$. Finally, observe that for canonical graphs 1-monotonicity and full 1-monotonicity coincide, due to the continuity of $f_{l, r}$.

To sum up, the projection operator $\sim$ associates to a 1-monotone (conservative) graph $\mathcal{H}$ some particular canonical 1-monotone (conservative) graph $\widetilde{\mathcal{H}}$. It can be checked that $\sim$ is a projection, namely, $\sim{ }^{\sim} \sim{ }^{\sim}$. However, further characterizations of the projected ICC graph $\widetilde{\mathcal{H}}$ should be developed in order to say in which precise sense it could be seen as the closest among all canonical graphs to the graph $\mathcal{H}$ (a property of this kind is expected in view of the results of [21] on projection of BC graphs).

Now, according to the idea explained in the introduction and by analogy with the boundary-value problems of $\S 4.1$, we will say that a solution $u$ of (1),(4) (in the sense of $\S 1.1$ ) satisfies the interface coupling condition (ICC) prescribed by a 1-monotone graph $\mathcal{H}$ if the defined hereabove traces $u_{l, r}(\cdot)$ and $F_{l, r}(\cdot)$ of the solution and of the flux, respectively, fulfill

$$
\text { for a.e. } t>0\left(u_{l}(t), u_{r}(t) ; F_{l}(t), F_{r}(t)\right) \in \widetilde{\mathcal{H}} \text {. }
$$

This represents a relaxation of the overdetermined condition $\left(u_{l}(t), u_{r}(t) ; F_{l}(t), F_{r}(t)\right) \in \mathcal{H}$, in the spirit of the celebrated Bardos-LeRoux-Nédélec condition. By analogy with the BLN kind interpretation of boundary-value problems, we will say that $\mathcal{H} \in \mathfrak{H}$ is a formal ICC graph, while the corresponding projection $\widetilde{\mathcal{H}} \in \widetilde{\mathfrak{H}}$ is the associated effective ICC graph.

Let us establish a simple relation between canonical 1-monotone graphs (in the conservative case) and the (conservative) $L^{1}$-dissipative germs. This will also provide a first, trivial though fundamental example of ICC graph $\mathcal{H}$. We have

Proposition 4.3. The following relations exist between $L^{1}$-dissipative germs and canonical ICC graphs. 
- Assume $\mathcal{G}$ is an $L^{1}$-dissipative germ. Then

$$
\mathcal{H}_{\mathcal{G}}:=\left\{\left(u_{l}, u_{r} ; f_{l}\left(u_{l}\right),-f_{r}\left(u_{r}\right)\right) \mid\left(u_{l}, u_{r}\right) \in \mathcal{G}\right\}
$$

is a canonical conservative 1-monotone graph in $\mathbb{R}^{2} \times \mathbb{R}^{2}$, i.e., $\mathcal{H}_{\mathcal{G}} \in \widetilde{\mathfrak{H}}_{0}$.

Similarly, if $\mathcal{G}$ is a non-conservative $L^{1}$-dissipative germ, then $\mathcal{H}_{\mathcal{G}} \in \widetilde{\mathfrak{H}}$.

- Reciprocally, every element $\mathcal{H}$ of $\widetilde{\mathfrak{H}}_{0}$ (respectively, of $\widetilde{\mathfrak{H}}$ ) has the form $\mathcal{H}_{\mathcal{G}}$ for the $L^{1}$-dissipative (respectively, non-conservative $L^{1}$-dissipative) germ $\mathcal{G} ; \mathcal{G}$ is equal to the domain of $\mathcal{H}$ seen as a map $\mathbb{R}^{2} \rightarrow 2^{\mathbb{R}^{2}}$.

- Moreover, $\mathcal{H}$ is maximal among all conservative (resp., among all) canonical 1-monotone graphs if and only if $\mathcal{G}$ is maximal among all (respectively, among all non-conservative) $L^{1}$-dissipative germs.

The justification of Proposition 4.3 is straightforward, based on the fact that for canonical ICC graphs there holds (with the notation $q^{l, r}$ for the Kruzhkov entropy fluxes, see $\S 1.2$ ):

$$
\left[u_{l, r}-\hat{u}_{l, r}, F_{l, r}-\hat{F}_{l, r}\right] \equiv q_{l, r}\left(u_{l, r}, \hat{u}_{l, r}\right) \text { whenever }\left(u_{l}, u_{r} ; f_{l}\left(u_{l}\right),-f_{r}\left(u_{r}\right)\right) \in \mathcal{H} \in \widetilde{\mathcal{H}} .
$$

Remark 4.4. Because of Proposition 4.3, existence and uniqueness of solutions - in the sense of $\S 1.1)-$ of (1),(4),(2) which are admissible in the sense of $\left(\mathrm{ADM}_{\mathcal{H}}\right)$ follow readily from the corresponding results for $\mathcal{G}$-entropy solutions, where $\mathcal{G}$ is the domain of the projected germ $\widetilde{\mathcal{H}}$.

Let us stress that we do not claim in this note that solutions in the above sense can be obtained by some reasonable approximation procedure constructed starting from the formal ICC graph $\mathcal{H}$. This is however known (see $\S 3.3$ ) for the particular case of ICC graphs determined by a transmission map: this will be our first non-trivial example in $\S 4.3$.

We do not claim neither that $\widetilde{\mathcal{H}}$ is a maximal canonical 1-monotone graph whenever $\mathcal{H}$ is a maximal 1monotone graph (however, this would be a natural property to think of and it is true for the examples given below in $\S 4.3$ ). Let us observe that such property would not be particularly informative ${ }^{13}$. Indeed, in order to clearly assess the approach to admissibility by ICC graphs, one needs to design some approximation procedure which does not involve the effective ICC graph $\widetilde{\mathcal{H}}$ but only the formal ICC graph $\mathcal{H}$, and that lead to solutions admissible in the sense $\left(\mathrm{ADM}_{\mathcal{H}}\right)$. Maximality of $\mathcal{H}$ would be required for existence of the so constructed approximate solutions; the fact that $\widetilde{\mathcal{H}}$ contains sufficiently many elements would be a consequence of convergence of such hypothetic approximation schemes. These questions are left for further research.

\subsection{Examples of interface coupling conditions}

In addition to the above relations between ICC and $L^{1}$-dissipative germs, let us provide three non-trivial examples where the formulation of ICC can be related to modeling assumptions taken on the interface.

\subsubsection{ICC determined by a transmission maps}

Let $\gamma \subset \mathbb{R} \times \mathbb{R}$ be a transmission map, i.e., a maximal monotone graph linking autorized couples of desired trace values $\left(\bar{u}_{l}, \bar{u}_{r}\right)$ on the interface. We can also take a monotone dissipation map $\psi: \gamma \rightarrow \mathbb{R}$.

Then, we define the associated interface coupling condition with the graph

$$
\mathcal{H}:=\left\{\left(u_{l}, u_{r} ; F_{l}, F_{r}\right) \in \mathbb{R}^{2} \times \mathbb{R}^{2} \mid\left(u_{l}, u_{r}\right) \in \gamma, F_{l, r} \in \mathbb{R} \text { and } F_{r}-F_{l}=\psi\left(u_{l}, u_{r}\right)\right\} .
$$

It is immediate to check that $\mathcal{H}$ is 1 -monotone (notice that it is fully 1-monotone only if $\gamma$ can be assimilated to the graph of a strictly increasing continuous function). It is also easy to see that $\mathcal{H}$ is maximal 1-monotone.

\footnotetext{
${ }^{13}$ This corresponds to our experience with justification of maximality of various $L^{1}$-dissipative germs. Most often, such justification is obtained using the proposition "a complete $L^{1}$-dissipative germ $\mathcal{G}$ is a maximal $L^{1}$-dissipative germ": i.e., maximality of $\mathcal{G}$ is usually not something that is checked beforehand, but it is a property which can be seen as a consequence of existence of $\mathcal{G}$-entropy solutions constructed by a suitable approximation procedure
} 
The meaning of this ICC is precisely the one expected from the description of transmission maps in $\S 3$. For instance, in the conservative case $\psi \equiv 0$, the pointwise condition (7) with the ICC graph $\mathcal{H}$ given by $(50)$ means that $\left(u_{l}(t), u_{r}(t)\right)$ are expected to take values in $\gamma$, while the value of the flux (equal to $F_{l}(t)$ and to $-F_{r}(t)$ due to the conservativity) is left arbitrary: this is precisely the relations $(23),(24)$.

\subsubsection{Interface coupling condition for limited-flux LWR model}

Let us consider one concrete example where the transmission map approach was only partly successful. Consider $f_{l} \equiv f_{r}$ defined by $f_{l, r}(u)=V_{\max } u(1-u)$ for $u \in[0,1]$ and extended by zero outside the interval [0,1]. Then equation (1) with the flux (4) (which turns out to be continuous) is a classical LWR model for road traffic. Colombo and Goatin in [41] introduced the conservative model based on LWR, but where the point $x=0$ played the role of an embedded interface. Indeed, it was required (withe the notation of the previous sections) that the flux traces $F_{l}=-F_{r}=f_{l, r}\left(u_{l, r}\right)$ at $\{x=0\}$ be upper bounded by a given function of time, that we will call $\mathrm{F}(\cdot)$ (this model was already mentioned in $\S 1.3)$. For the sake of simplicity, consider $\mathrm{F}(\cdot) \equiv$ const $\in\left(0, V_{\max } / 4\right)$. In this case, the underlying $L^{1}$-dissipative germ that we denote $\mathcal{G}_{\mathrm{F}}$ was described in [12].

In $[11, \S 4.5]$ we proposed a transmission map that corresponds to this model; however, among infinitely many suitable maps the one we fixed appeared is a particularly simple but not a particularly natural choice. The ICC of $\S 4.3 .1$ defined using the transmission map of [11, $\S 4.5]$ would be a suitable interface coupling condition for the model of [41]. However, in order to provide a more natural ICC for the same model, let us consider the graph in $\mathbb{R}^{2} \times \mathbb{R}^{2}$ given by

$$
\mathcal{H}^{\mathrm{F}}:=\{(u, u ; F,-F) \mid u \in[0,1], F \in[0, \mathrm{~F}]\} \bigcup\{(v, w ; \mathrm{F},-\mathrm{F}) \mid 0 \leq w \leq v \leq 1\} .
$$

This ICC simply means that we have two choices for the interface coupling. First, the transmission map Id (which, as we know, leads to Kruzhkov admissible solutions in the case $f_{l}=f_{l}$ ) can be used for the flux values below the critical level F. Second, non-physical (w.r.t. the non-constrained LWR model, where the car density $u$ cannot have decreasing jumps) shocks are authorized at the flux level F.

By a straightforward case study, we see that $\mathcal{H}^{\mathrm{F}}$ is a 1-monotone conservative graph in $\mathbb{R}^{2} \times \mathbb{R}^{2}$. As explained at the end of $\S 4.2$, we do not investigate about the maximality of $\mathcal{H}^{\mathrm{F}}$ not only because it is a relatively heavy task, but also because it is not useful: simpler information is available for the projected germ $\widetilde{\mathcal{H}^{F}}$. Indeed, it is not difficult to see that the $L^{1}$-dissipative germ which is the domain of $\widetilde{\mathcal{H}^{F}}$ contains all the couples that were identified as belonging to the germ $\mathcal{G}_{\mathrm{F}}$ : these couples correspond to all Kruzhkov admissible shocks at flux levels not exceeding $F$ and to the unique stationary non-classical shock precisely at the flux level $F$. Because we know that the domain of $\widetilde{\mathcal{H}^{F}}$ is an $L^{1}$-dissipative germ and in addition, we know from [12] that $\mathcal{G}_{\mathrm{F}}$ is a maximal $L^{1}$-dissipative germ, we conclude that they coincide. With the notation of Proposition 4.3, we conclude that $\widetilde{\mathcal{H}^{\mathrm{F}}}=\mathcal{H}_{\mathcal{G}_{\mathrm{F}}}$. In particular, the effective ICC graph $\widetilde{\mathcal{H}^{\mathrm{F}}}$ corresponding to the formal graph $\mathcal{H}^{\mathrm{F}}$ is a maximal canonical 1-monotone graph in $\mathbb{R}^{2} \times \mathbb{R}^{2}$.

To conclude the discussion of this example, let us make two observations. First, the above recipe works also in the case where the flux $f_{l, r}$ is not unimodal but has several extrema (see [39]). The ICC graph (51) encodes the same admissibility condition as the transmission map constructed in [11] (see [11, Fig. 1]). Second, recalling that in general, $\mathrm{F}$ is a $t$-dependent function one gets here a natural example of inhomogeneous ICC given by the family of graphs $\left(\mathcal{H}^{\mathrm{F}(t)}\right)_{t>0}$.

\subsubsection{Inflow-outflow conditions of the Robin type}

Finally, let us give another example that may make sense in applications. Consider a couple of monotone continuous functions $A_{l, r}: \mathbb{R} \rightarrow \mathbb{R}\left(\right.$ e.g., $A_{l, r}(u)=\frac{\lambda_{l, r}}{1-\lambda_{l, r}}(u)$ for some parameters $\left.\lambda_{l, r} \in(0,1)\right)$. Define

$$
\mathcal{H}:=\left\{\left(u_{l}, u_{r} ; F_{l}, F_{r}\right) \in \mathbb{R}^{2} \times \mathbb{R}^{2} \mid u_{l, r} \in \mathbb{R},-F_{r}=F_{l}=A_{l}\left(u_{l}\right)-A_{r}\left(u_{r}\right)\right\}
$$


This is a conservative ICC which prescribes the interface flux (from the left to the right) as the difference of inflow of the Robin kind (inflow at rate $A\left(u_{l}\right)$ if the left trace solution value is $u_{l}$ ) and of the outflow of the same kind (outflow at rate $A\left(u_{r}\right)$ if the right trace solution value is $u_{r}$ ). Thus, in this example we have two Robin boundary-value problems coupled by a global conservation property (to be compared with (22), where we have two Dirichlet problems coupled via a transmission map).

The 1-monotonicity of $\mathcal{H}$ given by (52) is checked by a straightforward calculation. Provided $u_{l} \neq \hat{u}_{l}$ and $u_{r} \neq \hat{u}_{r}$, given $\left(u_{l}, u_{r} ; F_{l}, F_{r}\right),\left(\hat{u}_{l}, \hat{u}_{r} ; \hat{F}_{l}, \hat{F}_{r}\right) \in \mathcal{H}$ we have due to the monotonicity of $A_{l, r}$ :

$$
\begin{aligned}
{\left[u_{l}-\hat{u}_{l}, F_{l}-\hat{F}_{l}\right]+\left[u_{r}-\hat{u}_{r}, F_{r}-\hat{F}_{r}\right]=} & \left|A_{l}\left(u_{l}\right)-A_{l}\left(\hat{u}_{l}\right)\right|+\left|A_{r}\left(u_{r}\right)-A_{r}\left(\hat{u}_{r}\right)\right| \\
& +\operatorname{sign}\left(u_{l}-\hat{u}_{l}\right)\left(A_{r}\left(u_{r}\right)-A_{r}\left(\hat{u}_{r}\right)\right)+\operatorname{sign}\left(u_{r}-\hat{u}_{r}\right)\left(A_{l}\left(u_{l}\right)-A_{l}\left(\hat{u}_{l}\right)\right) \geq 0 .
\end{aligned}
$$

The restrictions $u_{l} \neq \hat{u}_{l}$ and $u_{r} \neq \hat{u}_{r}$ are dropped using the upper semicontinuity of the bracket (47). The maximality of $\mathcal{H}$ can also be easily checked.

\subsection{On the interest of the ICC formalism}

To sum up, in this section we proposed the admissibility conditions of the form $\left(\mathrm{ADM}_{\mathcal{H}}\right)$ as a natural remedy to the non-uniqueness situation (NU). Indeed, the generality of ICC formulations shed light on the intrinsic non-uniqueness of solutions to discontinuous-flux problem (without prescribing an ICC, these problem become under-determined); and we hope that it can be helpful in modelling practice. Indeed, given an applied problem, it is not an easy task to point out the appropriate family of adapted entropy inequalities or the underlying germ. In contrast, at least in some cases the ICC behind a given physical situation can be easily identified from heuristic considerations (cf. $\S 4.3 .2$ for one example). Paying attention to interface coupling conditions relevant for a given model, much as one takes care of boundary conditions while considering PDEs in a bounded domain, may help to avoid hidden assumptions, to identify the appropriate coupling and in particular, to deduce the underlying germs and adapted entropy inequalities. As we already know, germs and adapted entropy inequalities are convenient technical tools that permit to assess well-posedness. At the same time, as shows the example of transmission maps, natural and relatively simple numerical schemes can be derived as soon as the underlying ICC is identified.

\section{APPENDIX: MEASURABLE FAMILIES OF GERMS}

A parametrized family of germs $\mathcal{G}(\cdot)$ underlying the notion of $\mathcal{G}(\cdot)$-entropy solution for discontinuous-flux problems with germs variable along interface $\Sigma$ cannot be taken completely arbitrary, because of measurability issues. Because the map $\sigma \in \Sigma \mapsto G(\sigma) \in 2^{\mathbb{R} \times \mathbb{R}}$ is a set-valued map, defining its measurability is not straightforward. In order to avoid too strong and difficult-to-verify measurability requirements (such as, e.g., the requirement that for all $\left(k_{l}, k_{r}\right) \in \mathbb{R} \times \mathbb{R}$, the characteristic function of the set $\left\{\sigma \in \Sigma \mid\left(k_{l}, k_{r}\right) \in \mathcal{G}(\sigma)\right\}$ be measurable) let us provide a notion of measurability suitable for the use which is made of subsets $\mathcal{G}(\sigma)$ of $\mathbb{R} \times \mathbb{R}$ in analysis of discontinuous-flux conservation laws. It is easily seen that measurability is most natural for maximal $L^{1}$-dissipative germs, therefore we will limit our attention to the case where $\mathcal{G}(\sigma)$ is maximal for a.e. $\sigma \in \Sigma$ ("a.e." being taken with respect to the natural Hausdorff measure on $\Sigma$ ). As in $\S 1.3$, for the sake of simplicity of the presentation let us limit the exposition to the case of the model one-dimensional problem with fluxes (4) but with a family $(\mathcal{G}(t))_{t>0}$ of maximal $L^{1}$-dissipative germs associated with $f_{l, r}$ in $(4)$. In this case, $\Sigma=\{t>0\}$ and the fluxes $f_{l, r}(\cdot)$ with which the germ $\mathcal{G}(t)$ is associated do not depend on $t$. Extension of the below Definition 4.5 and Proposition 4.6 to the general case is straightforward, up to heavy notational technicalities.

Our main tool is the "topology" on germs suggested in [15, formula (3.14)]. We use it to define open neighbourhoods of germs. Namely, given a number $\rho>0$ and a germ $\mathcal{G}$ associated with a couple of continuous scalar 
functions $f_{l, r}$ (with the corresponding Kruzhkov entropy fluxes denoted by $q_{l, r}(\cdot, \cdot)$ ), we set

$$
\mathfrak{O}_{\rho}(\mathcal{G})=\left\{\left(c_{l}, c_{r}\right) \in \mathbb{R} \times \mathbb{R} \mid \sup _{\left(a_{l}, a_{r}\right) \in \mathcal{G}}\left(q_{r}\left(c_{r}, a_{r}\right)-q_{l}\left(c_{l}, a_{l}\right)\right)<\rho\right\} .
$$

Clearly, for all $\rho>0, \mathfrak{O}_{\rho}(\mathcal{G})$ is an open subset of $\mathbb{R} \times \mathbb{R}$. In particular, the countable set $\mathbb{Q}^{2} \cap \mathfrak{O}_{\rho}(\mathcal{G})$ is always dense in $\mathfrak{O}_{\rho}(\mathcal{G})$. If $\rho_{1}<\rho_{2}$, then $\mathfrak{O}_{\rho_{1}}(\mathcal{G}) \subset \mathfrak{O}_{\rho_{2}}(\mathcal{G})$; furthermore, we have

$$
\bigcap_{n \in \mathbb{N}} \mathfrak{O}_{1 / n}(\mathcal{G})=\left\{\left(c_{l}, c_{r}\right) \in \mathbb{R} \times \mathbb{R} \mid \sup _{\left(a_{l}, a_{r}\right) \in \mathcal{G}}\left(q_{r}\left(c_{r}, a_{r}\right)-q_{l}\left(c_{l}, a_{l}\right)\right) \leq 0\right\} \equiv \mathcal{G}^{*}
$$

in the sequel, we assume that $\mathcal{G}$ is maximal and therefore we can write $\mathcal{G}$ for $\mathcal{G}^{*}$. Thus the family $\mathfrak{O}_{\rho}(\mathcal{G}), \rho>0$, is a family of open neighbourhoods of $\mathcal{G}$ shrinking to $\mathcal{G}$ as $\rho$ decreases to zero.

Definition 4.5. A family of germs $\mathcal{G}(\cdot)$ on $\Sigma$ is called measurable, if for all $\left(k_{l}, k_{r}\right) \in \mathbb{R} \times \mathbb{R}$ and all $\rho>0$, the set

$$
\left\{t \in \Sigma \mid\left(k_{l}, k_{r}\right) \in \mathfrak{O}_{\rho}(\mathcal{G}(t))\right\}
$$

is measurable (w.r.t. the standard one-dimensional Hausdorff measure on $\Sigma=\{(t, x) \mid t>0, x=0\}$ ).

The above notion of measurability, although it is probably not the ultimate one, allows for the following proposition which is crucial for defining $\mathcal{G}(\cdot)$-entropy solutions. For the sake of simplicity, we state it for the case of globally Lipschitz flux functions.

Proposition 4.6. Assume that $f_{l, r}$ are Lipschitz continuous with Lipschitz constant L. Assume that $\mathcal{G}(\cdot):=$ $(\mathcal{G}(t))_{t>0}$ is a measurable family of maximal $L^{1}$-dissipative germs. For every $\left(k_{l}, k_{r}\right) \in \mathbb{R} \times \mathbb{R}$, define the "remainder function"

$$
t \mapsto R_{\mathcal{G}(t)}\left(\left(k_{l}, k_{r}\right)\right):=\inf _{n \in \mathbb{N}}\left(\frac{1}{n}+2 L \inf _{\left(c_{l}, c_{r}\right) \in \mathfrak{O}_{1 / n}(\mathcal{G}(t))}\left(\left|k_{l}-c_{l}\right|+\left|k_{r}-c_{r}\right|\right)\right) .
$$

Then $R_{\mathcal{G}(\cdot)}\left(\left(k_{l}, k_{r}\right)\right)$ is a measurable function on $\Sigma=\{t>0\}$.

Proof. By definition, the characteristic function $\mathbb{1}_{\left(k_{l}, k_{r}\right) \in \mathfrak{O}_{1 / n}(\mathcal{G}(\sigma))}$ of the set given in (55) is measurable on $\Sigma$, for all $\left(k_{l}, k_{r}\right)$. Now notice that, $\mathfrak{O}_{1 / n}(\mathcal{G}(\sigma))$ being open, in the expression $\inf _{\left(c_{l}, c_{r}\right) \in \mathfrak{O}_{1 / n}(\mathcal{G}(\sigma))}\left(\left|k_{l}-c_{l}\right|+\left|k_{r}-c_{r}\right|\right)$ we can restrict the choice of $\left(c_{l}, c_{r}\right)$ to the countable set $\mathbb{Q}^{2} \cap \mathfrak{O}_{1 / n}(\mathcal{G}(\sigma))$. It is easy to check that

$\inf _{\left(c_{l}, c_{r}\right) \in \mathfrak{O}_{1 / n}(\mathcal{G}(t))}\left(\left|k_{l}-c_{l}\right|+\left|k_{r}-c_{r}\right|\right) \equiv \lim _{M \rightarrow+\infty} \inf _{\left(c_{l}, c_{r}\right) \in \mathbb{Q}^{2}}\left(\left(M+1-M \mathbb{1}_{\left(c_{l}, c_{r}\right) \in \mathfrak{O}_{1 / n}(\mathcal{G}(\sigma))}\right)\left(\left|k_{l}-c_{l}\right|+\left|k_{r}-c_{r}\right|\right)\right)$.

The measurability of $t \mapsto R_{\mathcal{G}(t)}\left(\left(k_{l}, k_{r}\right)\right)$ is now straightforward: we have just seen that $R_{\mathcal{G}(\cdot)}$ is obtained from measurable functions by inf (on countable sets) and lim operations.

Remark 4.7. The remainder function $R_{\mathcal{G}(\cdot)}((\cdot, \cdot))$ defined by (56) has all the properties required in $\S 1.3$. Indeed, in addition to the measurability proved in Proposition 4.6, this remainder function fulfills both (13) and (14). The justification of (14) is analogous to the calculation given in [15, page 43] (this is where the additive term $\frac{1}{n}$ in the definition (56) plays a role). The justification of (13) may require some a priori integrability assumption on $R_{\mathcal{G}(\cdot)}\left(\left(k_{l}, k_{r}\right)\right)$.

The properties of Proposition 4.6 and Remark 4.7 make the remainder functions defined by (56) exploitable in adapted entropy inequalities (12), see $\S 1.3$.

To conclude this Appendix, let us point out that the "vanishing viscosity" family of germs used in the work [14] of K.H. Karlsen, N.H. Risebro and the author is measurable in the sense of Definition 4.5. The proof is omitted; 
it is rather straightforward, starting from a piecewise constant approximation of the maps $\sigma \mapsto f_{l, r}(\sigma ; \cdot) \in C(\mathbb{R})$, where $f_{l, r}(\sigma, \cdot)$ are the one-sided traces at $\sigma \in \Sigma$ of the normal component of the vector-valued fluxes $\mathfrak{f}(t, x ; \cdot)$.

Acknowledgement. The author warmly thanks the direction and the personnel of IRMAR (University of Rennes) for their hospitality during the preparation of this paper.

\section{REFERENCES}

[1] Adimurthi, R. Dutta, S. S. Ghoshal, and G.D. Veerappa Gowda. Existence and nonexistence of TV bounds for scalar conservation laws with discontinuous flux. Comm. Pure Appl. Math., 64(1):84-115, 2011.

[2] Adimurthi, J.Jaffré, and G. D. Veerappa Gowda. Godunov-type methods for conservation laws with a flux function discontinuous in space. SIAM J. Numer. Anal., 42(1):179-2008, 2004.

[3] Adimurthi, S. Mishra, and G. D. Veerappa Gowda. Optimal entropy solutions for conservation laws with discontinuous fluxfunctions. J. Hyperbolic Differ. Equ., 2(4):783-837, 2005.

[4] Adimurthi and G. D. Veerappa Gowda. Conservation law with discontinuous flux. J. Math. Kyoto Univ., 43:27-70, 2003.

[5] J. Aleksić and D. Mitrović. On the compactness for two dimensional scalar conservation law with discontinuous flux. Comm. Math. Sciences, 4:963-971, 2009.

[6] B. Andreianov. Dissipative coupling of scalar conservation laws across an interface: theory and applications. In Hyperbolic problems - theory, numerics and applications. Vol. 1, 123-135, Ser. Contemp. Appl. Math. CAM 17, Singapore, 2012.

[7] B. Andreianov, Ph. Bénilan and S.N. Kruzhkov. $L^{1}$ theory of scalar conservation law with continuous flux function. J. Funct. Anal., 171 (2000), no.1, pp.15-33.

[8] B. Andreianov, K. Brenner and C. Cancès. Approximating the vanishing capillarity limit of two-phase flow in multi-dimensional heterogeneous porous medium. ZAMP Zeitschr. Angew. Math. Mech. 94(7-8), pp.655-667, 2014.

[9] B. Andreianov and C. Cancès. Vanishing capillarity solutions of Buckley-Leverett equation with gravity in two-rocks' medium. Comput. Geosci., 17(3):551-572, 2013.

[10] B. Andreianov and C. Cancès. A phase-by-phase upstream scheme that converges to the right solution for countercurrent two-phase flow in two-rocks media. Comput. Geosci. 18(2):211-226, 2014.

[11] B. Andreianov and C. Cancès. On interface transmission conditions for conservation laws with discontinuous flux of general shape. J. Hyperbolic Differ. Equ., accepted (2015). Available as HAL preprint hal.archives-ouvertes.fr/hal-00940756 (2014).

[12] B. Andreianov, P. Goatin and N. Seguin. Finite volume schemes for locally constrained conservation laws. Numer. Math. 115:609-645, 2010.

[13] B. Andreianov and N. Igbida. On uniqueness techniques for degenerate convection-diffusion problems. Int. J. Dyn. Syst. Diff. Equ., 4:3-34, 2012.

[14] B. Andreianov, K. H. Karlsen and N. H. Risebro, On vanishing viscosity approximation of conservation laws with discontinuous flux, Netw. Heter. Media 5(3):617-633, 2010.

[15] B. Andreianov, K. H. Karlsen, and N. H. Risebro. A theory of $L^{1}$-dissipative solvers for scalar conservation laws with discontinuous flux. Arch. Ration. Mech. Anal., 201(1):27-86, 2011.

[16] B. Andreianov, F. Lagoutière, N. Seguin and T. Takahashi. Small solids in an inviscid fluid. Netw. Heterog. Media, 5(3):385-404, 2010.

[17] B. Andreianov, F. Lagoutière, N. Seguin and T. Takahashi. Well-posedness for a one-dimensional fluid-particle interaction model. SIAM J. Math. Anal., 46(2):1030-1052, 2014.

[18] B. Andreianov and M. Maliki. A note on uniqueness of entropy solu- tions to degenerate parabolic equations in $R^{N}$. NODEA Nonlinear Differ. Equ. Appl. 17, 109-118, 2010.

[19] B. Andreianov and D. Mitrović. Entropy conditions for scalar conservation laws with discontinuous flux revisited. Ann. Inst. H. Poincaré: Anal. Non Linéaire (C), 2014, appeared online.

[20] B. Andreianov and K. Sbihi. Scalar conservation laws with nonlinear boundary conditions. C. R. Acad. Sci. Paris, Ser. I 345:431-434, 2007.

[21] B. Andreianov and K. Sbihi. Well-posedness of general boundary-value problems for scalar conservation laws. Trans. AMS, to appear (2015). Available as HAL preprint hal.archives-ouvertes.fr/hal-00708973

[22] B. Andreianov and N. Seguin. Well-posedness of a singular balance law. Discr. Cont. Dyn. Syst. A 32(6):1939-1964, 2012.

[23] F. Andreu, N. Igbida, J.M. Mazón and J. Toledo. $L^{1}$ existence and uniqueness results for quasi-linear elliptic equations with nonlinear boundary conditions. Ann. Inst. H. Poincaré (C) Anal. Non Linéaire 24(1):61-89, 2007.

[24] E. Audusse and B. Perthame. Uniqueness for scalar conservation laws with discontinuous flux via adapted entropies. Proc. Roy. Soc. Edinburgh A, 135(2):253-265, 2005.

[25] F. Bachmann and J. Vovelle. Existence and uniqueness of entropy solution of scalar conservation laws with a flux function involving discontinuous coefficients. Comm. Partial Differential Equations, 31:371-395, 2006.

[26] C. Bardos, A. Y. le Roux, and J.-C. Nédélec. First order quasilinear equations with boundary conditions. Comm. Partial Differential Equations, 4(9):1017-1034, 1979.

[27] P. Bénilan. Equations d'évolution dans un espace de Banach quelconques et applications. Thèse d'état, 1972. 
[28] P. Bénilan, J. Carrillo, and P. Wittbold. Renormalized entropy solutions of scalar conservation laws. Ann. Scuola Norm. Sup. Pisa Cl. Sci. (4), 29(2):313-327, 2000.

[29] P. Bénilan and S. N. Kruzhkov. Conservation laws with continuous flux functions. NoDEA Nonlinear Differential Equations Appl., 3(4):395-419, 1996.

[30] R. Bürger, K. H. Karlsen, and J. D. Towers. An Engquist-Osher-type scheme for conservation laws with discontinuous flux adapted to flux connections. SIAM J. Numer. Anal., 47(3):1684-1712, 2009.

[31] F. Buzzi, M. Lenzinger, and B. Schweizer. Interface conditions for degenerate two-phase flow equations in one space dimension. Analysis, 29:299-316, 2009.

[32] J. Carrillo. Conservation laws with discontinuous flux functions and boundary condition. J. Evol. Equ. 3(2):283-301, 2003.

[33] C. Cancès. Finite volume scheme for two-phase flow in heterogeneous porous media involving capillary pressure discontinuities. M2AN Math. Model. Numer. Anal., 43(5):973-1001, 2009.

[34] C. Cancès. Asymptotic behavior of two-phase flows in heterogeneous porous media for capillarity depending only on space. I. Convergence to the optimal entropy solution. SIAM J. Math. Anal., 42(2):946-971, 2010.

[35] C. Cancès. Asymptotic behavior of two-phase flows in heterogeneous porous media for capillarity depending only on space. II. Non-classical shocks to model oil-trapping. SIAM J. Math. Anal., 42(2):972-995, 2010.

[36] C. Cancès. On the effects of discontinuous capillarities for immiscible two-phase flows in porous media made of several rocktypes. Networks Het. Media, 5(3):635-647, 2010.

[37] C. Cancès, T. Gallouët, and A. Porretta. Two-phase flows involving capillary barriers in heterogeneous porous media. Interfaces Free Bound., 11(2):239-258, 2009.

[38] C. Cancès and N. Seguin. Error estimate for Godunov approximation of locally constrained conservation laws. SIAM J. Numer. Anal., 50(6):336-360, 2012.

[39] C. Chalons, P. Goatin and N. Seguin. General constrained conservation laws. Application to pedestrian flow modeling. Netw. Heterog. Media 8(2):433-463, 2013.

[40] G. Chavent, G. Cohen and J. Jaffré. A Finite-Element simulator for incompressible two-phase flow. Transp. Porous Media 2: 465-478, 1987.

[41] R. M. Colombo and P. Goatin. A well posed conservation law with a variable unilateral constraint. J. Differential Equations, 234(2): 654-675, 2007.

[42] G. Crasta, V. De Cicco and G. De Philippis. Kinetic formulation and uniqueness for scalar conservation laws with discontinuous flux. Preprint arXiv:1404.5837, 2014.

[43] C. M. Dafermos. Hyperbolic conservation laws in continuum physics, volume 325 of Grundlehren der Mathematischen Wissenschaften [Fundamental Principles of Mathematical Sciences]. Springer-Verlag, Berlin, 3rd ed., 2010.

[44] S. Diehl. A uniqueness condition for nonlinear convection-diffusion equations with discontinuous coefficients. J. Hyperbolic Diff. Equ. 6(1):127-159, 2009.

[45] M. Karimou Gazibo. Etudes mathématiques et numériques des problèmes paraboliques avec des conditions aux limites. [French, English] Ph.D. thesis, Besançon, France, 2013.

[46] T. Gimse and N. H. Risebro. Solution of the Cauchy problem for a conservation law with a discontinuous flux function. SIAM J. Math. Anal., 23(3):635-648, 1992.

[47] P. Gwiazda, A. Świerczewska-Gwiazda, P. Wittbold and A. Zimmermann. Multi-dimensional scalar balance laws with discontinuous flux. J. Funct. Anal. 267(8):2846-2883, 2014.

[48] M. Bulíček, P. Gwiazda, J. Málek and A. Świerczewska-Gwiazda. On scalar hyperbolic conservation laws with a discontinuous flux. M3AS Math. Models Methods Appl. Sci. 21(1):89-113, 2001.

[49] M. Bulíček, P. Gwiazda and A. Świerczewska-Gwiazda. Multi-dimensional scalar conservation laws with fluxes discontinuous in the unknown and the spatial variable. M3AS Math. Models Methods Appl. Sci. 3:407-439, 2013.

[50] C. Imbert and R. Monneau. Flux-limited solutions for quasi-convex Hamilton-Jacobi equations on networks. Preprint, arXiv:1306.2428, 2013.

[51] K. H. Karlsen, N. H. Risebro, and J. D. Towers. $L^{1}$ stability for entropy solutions of nonlinear degenerate parabolic convectiondiffusion equations with discontinuous coefficients. Skr. K. Nor. Vidensk. Selsk. 3:1-49, 2003.

[52] S. N. Kruzhkov. First order quasilinear equations with several independent variables. Mat. Sb. (N.S.), 81 (123):228-255, 1970.

[53] S.N. Kruzhkov and E.Yu. Panov. Osgood's type conditions for uniqueness of entropy solutions to Cauchy problem for quasilinear conservation laws of the first order. Ann. Univ. Ferrara, V.15(1994), pp.31-53.

[54] Y.-S. Kwon and A. Vasseur. Strong traces for solutions to scalar conservation laws with general flux. Arch. Rational Mech. Anal., 185(3):495-513, 2007.

[55] F. Lagoutière, N. Seguin, and T. Takahashi. A simple $1 D$ model of inviscid fluid-solid interaction. J. Differ. Equ. 245(11):3503$3544,2008$.

[56] S. Martin and J. Vovelle. Convergence of implicit finite volume methods for scalar conservation laws with discontinuous flux function. M2AN Math. Model. Numer. Anal. 42(5):699-727, 2008.

[57] D. Mitrović. New Entropy Conditions for Scalar Conservation Laws with Discontinuous Flux. Discr. Cont. Dyn. Systems A, 30:1191-1210, 2011. 
[58] D. Mitrović. Proper entropy conditions for scalar conservation laws with discontinuous flux. arXiv preprint arXiv:1202.5853, 2012.

[59] E. Yu. Panov. On sequences of measure valued solutions for a first order quasilinear equation (Russian). Mat. Sb., 185(2):87106, 1994; Engl. tr. in Russian Acad. Sci. Sb. Math., 81(1):211-227, 1995.

[60] E. Yu. Panov. Existence of strong traces for generalized solutions of multidimensional scalar conservation laws. J. Hyperbolic Differ. Equ., 2(4):885-908, 2005.

[61] E. Y. Panov. Existence of strong traces for quasi-solutions of multidimensional conservation laws. J. Hyperbolic Differ. Equ., 4(4):729-770, 2007.

[62] E. Yu. Panov. On existence and uniqueness of entropy solutions to the Cauchy problem for a conservation law with discontinuous flux. J. Hyperbolic Differ. Equ., 6(3):525-548, 2009.

[63] B. Perthame. Kinetic formulation of conservation laws, volume 21 of Oxford Lecture Series in Mathematics and its Applications. Oxford University Press, Oxford, 2002.

[64] N. Seguin and J. Vovelle. Analysis and approximation of a scalar conservation law with a flux function with discontinuous coefficients. Math. Models Methods Appl. Sci., 13(2):221-257, 2003.

[65] A. Szepessy. Measure-valued solution of scalar conservation laws with boundary conditions. Arch. Rat. Mech. Anal., 107(2):182193, 1989.

[66] J. D. Towers. Convergence of a difference scheme for conservation laws with a discontinuous flux. SIAM J. Numer. Anal., 38(2):681-698, 2000. 